\title{
Hochschild homology and cohomology of Generalized Weyl algebras: the quantum case
}

\author{
Andrea Solotar \\ Mariano Suárez-Alvarez \\ Quimey Vivas *
}

June 27,2011

\begin{abstract}
We determine the Hochschild homology and cohomology of the generalized Weyl algebras of rank one which are of 'quantum' type in all but a few exceptional cases.

2010 MSC: 16E40, 16E65, 16U80, 16W50, 16W70.
\end{abstract}

\section{Introduction}

The Hochschild cohomology $\mathrm{HH}^{*}(\mathrm{~A})$ and homology $\mathrm{HH}_{*}(\mathrm{~A})$ of a k-algebra $\mathrm{A}$ are invariants which are usually hard to compute. For a long time it has been known that they are related to the smoothness of the algebra. For example, if $A$ is a commutative algebra $A$ essentially of finite type - i.e., a quotient of a polynomial algebra on a finite number of variables by an ideal, or a localization of one of these algebras — several authors [2] [5] [12] [14] [15] have obtained results which can be summarized in the statement

If $\mathrm{k}$ is a field, $\operatorname{gldim}(A)<\infty$ if and only if there exists $n$ such that $\mathrm{HH}_{\mathrm{i}}(\mathrm{A})=0$, for all $\mathrm{i}>\mathrm{n}$.

Some years ago, L. Avramov and S. Iyengar [1] proved a cohomological version of this property:

if $\mathrm{k}$ is a field, $\operatorname{gldim}(A)<\infty$ if and only if there exists $n$ such that $\mathrm{HH}^{\mathrm{i}}(\mathrm{A})=0$, for all $\mathrm{i}>\mathrm{n}$.

The non commutative case is different. After D. Happel asked in [11]

given a finite dimensional $k$-algebra $A$, is it true that the vanishing of $\mathrm{HH}^{i}(A)$ for all large $i$ implies that $\operatorname{gldim}(\mathrm{A})<\infty$ ?

several articles have been devoted to trying to provide an affirmative answer. However, in [8] a counterexample was given, the "small" algebra $k\langle x, y\rangle /\left(x^{2}, y^{2}, x y+q y x\right)$, with $q \in k^{*}$ not a root

${ }^{*}$ This work has been supported by the projects UBACYTX212, PIP-CONICET 112-200801-00487, PICT-2007-02182, UBACYT 20020090300102 IJ and MATHAMSUD-NOCOMALRET. The first and second authors are research members of CONICET (Argentina). A. Solotar thanks Universidad de Valparaíso (Project MECESUP UVA0806). 
of unity. Subsequently, Y. Han [10] showed that the Hochschild homology of this algebra does not vanish in infinitely many degrees, proposing thus what is now known as Han's conjecture:

If all the higher Hochschild homology groups of a finite dimensional algebra vanish, then the global dimension of the algebra is finite.

This conjecture has been proved to be true for commutative algebras essentially of finite type, not necessarily finite dimensional [2,5], for finite dimensional graded local algebras [7], for finite dimensional monomial algebras [10], for finite dimensional graded cellular algebras in characteristic zero [7], for finite dimensional Koszul algebras in characteristic zero [7], for quantum complete intersections [6], for finite dimensional graded local algebras satisfying the hypotheses of Theorem II of [17], and for algebras satisfying the hypotheses of Theorem I of [17].

The general answer is, however, still unknown. The proof of this last case makes use of the fact that Hochschild homology is functorial, which is not valid for Hochschild cohomology. The results of Theorem I of [17] led us to consider the conjecture without the hypothesis of $A$ being finite dimensional.

It is worth to notice that the proof of the conjecture - homological and cohomological - in the commutative case, uses the existence of a model, that is, a differential graded algebra quasiisomorphic to the inital one, and having thus isomorphic Hochschild homology and cohomology. The importance of the model, stated informally, is that it allows, in a certain way, to treat more easily the singularities of the algebra. In other words, the difficulty is no longer in the algebra itself, but in the differentials of the model. This kind of model, coming from algebraic topology, always exists in the commutative essentially of finite type case, but usually not in the non commutative case. One example of a situation where it exists is treated in Theorem II of [17]. Also, for Koszul algebras, it is clear that the complex which can be used to compute Hochschild (co)homology is similar to the one constructed from a model in the commutative case. So, in our opinion, and although the methods used in [7], [10], [6] are different, Han's conjecture has been proven, up to now, for algebras which have some kind of "model".

Following this point of view, in this article we prove it for a family of non commutative algebras $A_{q}$, the quantum generalized Weyl algebras, which we shall call simply Bavula algebras. For this we compute their Hochschild cohomology and homology, completing in this way the results of [9], leaving out only a few cases. We get the following two results:

Theorem 1.1. Let $A=A\left(\sigma_{q}, a\right)$ be a Bavula algebra with $q \in k^{\times}$not a root of 1 . Then

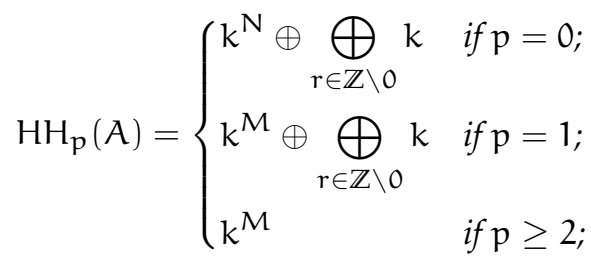

$$
\begin{aligned}
& H H^{p}(A)= \begin{cases}k & \text { if } p=0,1 \\
k^{N} & \text { if } p=2 \\
k^{M} & \text { if } p \geq 3\end{cases}
\end{aligned}
$$


where $\mathrm{N}=\operatorname{deg} \mathrm{a}$ and $\mathrm{M}=\operatorname{deg}\left(\mathrm{a}: \mathrm{a}^{\prime}\right)$.

Theorem 1.2. Let $\mathrm{A}=\mathrm{A}\left(\sigma_{\mathrm{q}}, \mathrm{a}\right)$ be a Bavula algebra with $\mathrm{q} \in \mathrm{k}^{\times}$such that $\mathrm{q}^{e}=1$. Then

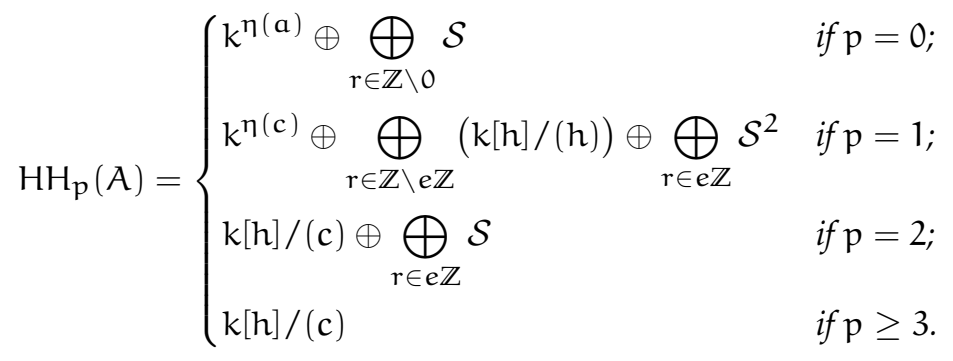

$$
\begin{aligned}
& H^{p}(A)= \begin{cases}\bigoplus_{r \in e \mathbb{Z}} \mathcal{S} & \text { if } p=0 ; \\
\bigoplus_{r \in e \mathbb{Z}} \mathcal{S}^{2} & \text { if } \mathrm{p}=1 ; \\
k^{\eta(a / c)} \oplus k[h] / c \oplus \bigoplus_{r \in e \mathbb{Z}} \mathcal{S} & \text { if } p=2 ; \\
k[h] / c & \text { if } p \geq 3 .\end{cases}
\end{aligned}
$$

where, for a polynomial $f \in k[h]$, we write $\eta(f)=\operatorname{deg} f-\frac{1}{e} \operatorname{deg} \mathcal{N}(f)$ with $\mathcal{N}$ the operator defined in section 2 below, $\mathrm{N}=\operatorname{deg} \mathrm{a}, \mathrm{c}=\left(\mathrm{a}: \mathrm{a}^{\prime}\right)$ and and $\mathrm{M}=\operatorname{deg} \mathrm{c}$.

Whether the 'quantum parameter' $q$ appearing in the definition of these Bavula algebras is a root of unity or not is a fact that plays a fundamental role, since the computations differ substantially in both cases.

The article is organized as follows. In Section 2 we fix the notations and state some auxiliar results that will be necessary in the rest of the article. In Section 3 we recall form [3] the definition of these algebras and we study their global dimension. In Section 4 we compute a projective resolution of our algebra $A$ as an A-bimodule. In Section 5 we compute the Hochschild homology and, finally, in Section 6 we compute the Hochschild cohomology.

\section{Notations and some generalities}

Let $k$ be a field of characteristic zero. If $\lambda \in k$ and $n \geq 0$, we write $[n]_{\lambda}=1+\lambda+\cdots+\lambda^{n-1}$; in particular, if $\lambda=1$, then $[n]_{\lambda}=n$.

We fix a scalar $q \in k \backslash\{0,1\}$ and a monic polynomial $a=\sum_{i=0}^{N} \alpha_{i} h^{i} \in k[h]$ of degree deg $a=$ $N>1$. Throughout the paper, $A=A(a, q)$ will denote the $k$-algebra freely generated by letters $y$, $h$ and $x$ subject to the relations

$$
x h=q h x, \quad y x=a(h), \quad \quad h y=q y h, \quad x y=a(q h) .
$$

It is easy to see that the $\operatorname{set}\left\{y^{i} h^{j}: i, j \geq 0\right\} \cup\left\{h^{j} x^{k}: j \geq 0, k \geq 1\right\}$ is a basis of $A$ as a $k$-module. 
We let $\sigma=\sigma_{\mathrm{q}}: k[\mathrm{~h}] \rightarrow k[\mathrm{~h}]$ be the algebra automorphism such that $\sigma(\mathrm{h})=\mathrm{qh}$. Then $\mathrm{xr}=\sigma(\mathrm{r}) \mathrm{x}$ and $r y=y \sigma(r)$ for all $r \in k[h]$, and $x y=\sigma(a)$. Moreover, the algebra $A$ is $\mathbb{Z}$-graded in such a way that the generators have degrees $|y|=1,|h|=0$ and $|x|=-1$; we refer to the degree of an element homogeneous with respect to this grading as its weight.

We remark that there is an algebra isomorphism $\Phi: A(a, q) \rightarrow A\left(\sigma_{q}(a), q^{-1}\right)$ such that $\Phi(x)=$ $y, \Phi(y)=x$ and $\Phi(h)=h$. This isomorphism maps the homogeneous component of weight $r \in \mathbb{Z}$ of $A(a, q)$ to the component of weight $-r$ of its codomain. This observation will allow us to carry out homological computations just in weights $r \geq 0$, since all arguments will be transferable to negative degrees using $\Phi$.

Given polynomials $p, t \in k[h]$, we shall write $(p: t)$ their greatest common divisor and $p^{\prime}$ the derivative of $p$ and we make the convention that the degree of the zero polynomial is $-\infty$.

We let $c=\left(a: a^{\prime}\right)$ and $M=\operatorname{deg}(c)$. If $q$ is a root of 1 , we let $e$ be its order; if $q$ is not a root of unity we let $e=0$. If $r \in \mathbb{Z}$, we say that $r$ is singular if $e \mid r$, and that it is regular otherwise.

The subring of $k[h]$ fixed by $\sigma$ is $S=\operatorname{ker}(\sigma-1)$, generated by $h^{e}$. We say that a polynomial $p \in k[h]$ is singular if $p \in \mathcal{S}$. More generally, when $e>0$ we have $\operatorname{ker}\left(\sigma-q^{l}\right)=h^{l} k\left[h^{e}\right]$ for each $l \in\{0, \ldots, e-1\}$.

If $e>0$, for each $f \in k[h]$ such that $f(0) \neq 0$ we define

$$
\mathcal{N}(f)=\operatorname{lcm}\left(f: \sigma(f): \cdots: \sigma^{e-1}(f)\right) \quad \text { and } \quad \bar{f}=\frac{\mathcal{N}(f)}{f} .
$$

Clearly $\sigma(\mathcal{N}(f))$ is a scalar multiple of $\mathcal{N}(f)$; evaluating both at 0 shows then they are in fact equal, so that $\mathcal{N}(f) \in \mathcal{S}$. The reason which motivates our interest in the operator $\mathcal{N}$ is the following proposition:

Proposition 2.1. Let $\mathrm{f}, \mathrm{g} \in \mathrm{k}[\mathrm{h}]$ and suppose $\mathrm{f}(0) \neq 0$.

(i) If $\mathrm{g} \in \mathcal{S}$, then $\overline{\mathrm{f}} \mid \mathrm{g}$.

(ii) If $\mathrm{g} \in \mathcal{S}$ and $\mathrm{f} \mid \mathrm{g}$, then there exists $\mathrm{s} \in \mathcal{S}$ such that $\mathrm{g}=\mathcal{N}(\mathrm{f}) \mathrm{s}$.

Proof. Since $f g \in \mathcal{S}$, we know that $\sigma^{i}(f g)=f g$, so $\sigma^{i}(f) \mid f g$ for all $i$. The first statement follows now from the definition of $\mathcal{N}(f)$. The second one is an immediate consequence.

We end this section with two technical lemmas which will be of use in the computation of Sections 5 and 6 .

Lemma 2.2. Let $\mathrm{f} \in \mathrm{k}[\mathrm{h}]$ and suppose that $\mathrm{f}(0) \neq 0$ and that $\mathrm{q}$ is a root of unity of order e. If $\pi: k[\mathrm{~h}] \rightarrow$ $\mathrm{k}[\mathrm{h}] /(\mathrm{f})$ be the canonical projection, then for each $\mathrm{l} \geq 0$ we have

$$
\operatorname{dim} \pi\left(h^{l} S\right)=\frac{\operatorname{deg} \mathcal{N}(f)}{e} .
$$

Proof. Since multiplication by $\pi(h)$ on $k[h] /(f)$ is an isomorphism, it is enough to prove this when $l=0$. Let us consider the following commutative diagram, in which the morphisms are the 
obvious ones:

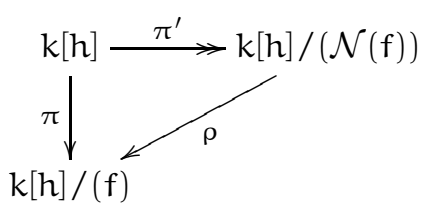

Let $G$ be a cyclic group of order e generated by an element $g \in G$. We endow $k[h]$ with the action of $G$ such that $g$ acts as $\sigma$. Since $\mathcal{N}(f)$ is $G$-invariant there is an induced action on $k[h] /(\mathcal{N}(f))$. The map $\pi^{\prime}$ is surjective, so the restriction $\left(\pi^{\prime}\right)^{\mathrm{G}}: \mathcal{S} \rightarrow(k[\mathrm{~h}] /(\mathcal{N}(\mathrm{f})))^{\mathrm{G}}$ is surjective too.

The situation is described by the following commutative diagram

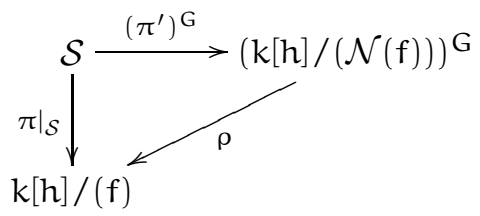

If $s \in \mathcal{S}$ is such that $\left.\pi\right|_{\mathcal{S}}(\mathrm{s})=0$, then there exists a $\mathrm{b} \in \mathrm{k}[\mathrm{h}]$ such that $\mathrm{fb}=\mathrm{s} \in \mathcal{S}$ and it follows from the previous proposotion that $b=\bar{f} s_{1}$ for some $s_{1} \in \mathcal{S}$ : we see that $s=\mathcal{N}(f) s_{1}$ and $\left(\pi^{\prime}\right)^{G}(s)=0$. As $\left(\pi^{\prime}\right)^{G}$ is surjective, this implies that the map $\rho$ is injective and, as a consequnce, that $\operatorname{dim} \pi(S)=$ $\operatorname{dim}(k[h] /(\mathcal{N}(f)))^{\mathrm{G}}$.

Now, $k[h] /(\mathcal{N}(f))$ has $\left\{h^{i}: 0 \leq i<\operatorname{deg} \mathcal{N}(f)\right\}$ as a basis and the action of $G$ is diagonal with respect to it. It is immediate, then, that $\operatorname{dim}(k[h] /(\mathcal{N}(f)))^{G}=\frac{1}{e} \operatorname{deg} \mathcal{N}(f)$

Lemma 2.3. Let $f \in k[h]$ such that $f(0) \neq 0, q \in k$ a root of unity of order $e, l \geq 0$ and consider the $\mathcal{S}$-linear map $\psi_{f, l}: p \in k[h] \mapsto\left(\sigma-q^{l}\right)(f p) \in k[h]$. Then

$\operatorname{coker} \psi_{f, l} \cong h^{l} \mathcal{S} \oplus k^{\eta(f)}$

with $\eta(f)=\operatorname{deg} f-\frac{1}{e} \operatorname{deg} \mathcal{N}(f)$.

Proof. We decompose $k[h] \cong \mathcal{S} \oplus h \mathcal{S} \cdots \oplus \mathrm{h}^{\mathrm{e}-1} \mathcal{S}$ as $\mathcal{S}$-module. Since $\operatorname{ker}\left(\sigma-\mathrm{q}^{\mathrm{l}}\right)=\mathrm{h}^{\mathrm{l}} \mathcal{S}$, the map $\sigma-q^{l}$ induces an injective map $k[h] / h^{l} \mathcal{S} \rightarrow k[h]$, still denoted $\sigma-q^{l}$. Consider the following diagram

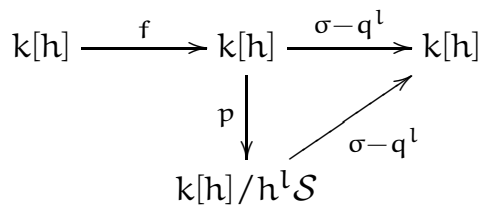

Because $\sigma-q^{l}$ is injective, it is immediate that $\operatorname{coker} \psi_{f, l} \cong h^{l} \mathcal{S} \oplus \operatorname{coker}(p \circ f)$ and, since

$$
\text { coker } p \circ f \cong \frac{k[h]}{h^{l} \mathcal{S}+(f)} \cong \frac{k[h]}{(f)} / \pi\left(h^{l} S\right)
$$

with $\pi$ the map defined in Lemma 2.2, we see that dim coker $p \circ f=\eta(f)$. 
We remark that the isomorphism in the statement of this lemma is actually an isomorphism of $\mathcal{S}$-modules, if we identify the summand $k^{\eta(f)}$ with the quotient $k[h] /\left(h^{l} \mathcal{S}+(f)\right)$ appearing in the proof.

\section{Global dimension}

Given a noetherian algebra $R$ which is an integral domain, a non zero central element $a \in R$ and an algebra automorphism $\sigma \in \operatorname{Aut}_{k}(R)$, the Bavula algebra $\Lambda=\Lambda(R, \sigma, a)$ is the k-algebra generated by $R$ and two variables $x, y$ subject to the relations

$$
y x=a, \quad x y=\sigma(a), \quad x r=\sigma(r) x, \quad r y=y \sigma(r)
$$

for all $r \in R$. It was introduced by V. Bavula in [3] with the name of generalized Weyl algebra. The algebra $A$ introduced in Section 2 is a special case of this construction.

The algebra $A$ is a noetherian domain and there is a $\mathbb{Z}$-grading on $\Lambda$ with all elements of $R$ in degree 0 , and $x$ and $y$ in degrees -1 and 1 , respectively; we denote $|u|$ the degree of an homogeneous element $u \in \Lambda$ and call it its weight.

Using the easily obtained description of automorphisms of $k[h]$, one can obtain the following classification of the algebras of the form $\Lambda(k[h], \sigma, a)$ up to isomorphism, as in [13]:

Proposition 3.1. The algebra $\Lambda=\Lambda(k[h], \sigma, a)$ is isomorphic to exactly one of the following list:

1. $\Lambda(k[h], I d, a)$ for some $a \in k[h]$;

2. $\Lambda\left(k[h], \sigma_{c l}, a\right)$ with $\sigma_{c l}(h)=h-1$ and $a \in k[h]$;

3. $\Lambda\left(k[h], \sigma_{\mathrm{q}}, \mathrm{a}\right)$ with $\mathrm{q} \in \mathrm{k} \backslash\{0,1\}, \sigma_{\mathrm{q}}(\mathrm{h})=\mathrm{qh}$ and $\mathrm{a} \in \mathrm{k}[\mathrm{h}]$.

We refer to case 2 as the classical case and to case 3 as the quantum case.

If $\mathrm{b} \in \mathrm{R}$, let $\mathrm{I}(x, \mathrm{~b})=\Lambda x+\Lambda \mathrm{b} \subseteq \Lambda$. Bavula proved in [4] the following result concerning the global dimension of his algebras:

Theorem 3.2. [4, Thm. 3.5] If $\mathrm{R}$ is a commutative Noetherian domain of finite global dimension $\mathrm{n}$ and $a \neq 0$, then the following two conditions are equivalent:

- $\operatorname{gldim} \Lambda<\infty$

- $\operatorname{pdim}_{\wedge} \wedge / \mathrm{I}(x, \mathfrak{p})<\infty$ for all prime ideals $\mathfrak{p}$ of $\mathrm{R}$ which contain $\mathrm{a}$.

When $R=k[h]$, the hypotheses of this theorem are satisfied and we can give a characterization of Bavula algebras of finite global dimension.

Theorem 3.3. Let $R=k[h], a \in R, \sigma \in \operatorname{Aut}_{k}(R)$ and $\Lambda=\Lambda(R, \sigma, a)$. Then

$$
\operatorname{gldim} \wedge<\infty \Longleftrightarrow\left(a: a^{\prime}\right)=1 \text {. }
$$

Proof. The "only if" part has been proved by Bavula in [4], so we only have to prove the "if" part. 
Let $p \in R$ be a prime element which divides $a$, so that there is $a b \in R$ with $a=p b$. The canonical short exact sequence of left $\Lambda$-modules

$$
0 \rightarrow \mathrm{I}(x, \mathrm{p}) \rightarrow \Lambda \rightarrow \Lambda / \mathrm{I}(\mathrm{x}, \mathrm{p}) \rightarrow 0
$$

tells us that $\operatorname{pdim}_{\wedge} \wedge / I(x, p)<\operatorname{pdim}_{\wedge} I(x, p)+2$. We shall prove that if $\left(a: a^{\prime}\right)=1$, then $I(x, p)$ is a projective $\Lambda$-module.

We start by showting that $\Lambda x \cap \wedge p=I(x, b) p$. Fix $f \in \Lambda x \cap \wedge p$; we may assume that $f$ is homogeneous with respect to the weight and that $|f|=r \geq 0$ : the case in which the weight of $f$ is negative is similar. Since $f \in \Lambda x \cap \wedge p$, there exist $u, v \in R$ such that $f=y^{r+1} u x=y^{r} v p$. As

$$
y^{r+1} u x=y^{r} a \sigma^{-1}(u)=y^{r} p b \sigma^{-1}(u)=y^{r} \sigma^{-1}(u) b p
$$

and $\Lambda$ is a domain, $\sigma^{-1}(u) b=v$ and, in consequence, $f \in I(x, b) p$. The other inclusion is easy.

Consider now the short sequence of left $\Lambda$-modules

$$
0 \longrightarrow \mathrm{I}(\mathrm{x}, \mathrm{b}) \stackrel{\gamma}{\longrightarrow} \wedge \oplus \wedge \stackrel{\phi}{\longrightarrow} \mathrm{I}(\mathrm{x}, \mathrm{p}) \longrightarrow 0
$$

where $\phi(\alpha, \beta)=\alpha x-\beta p$ and $\gamma(w)=\left(w p x^{-1}, w\right)$; this last expression makes sense because for all $p \in I(x, b)$ we have $w p \in A x=x A$ and $A$ is a domain.

It is clear that $\gamma$ is a monomorphism, $\phi$ is an epimorphism and that $\operatorname{im} \gamma \subseteq \operatorname{ker} \phi$. The sequence (1) is in fact exact: to check the other inclusion suppose that $(\alpha, \beta) \in \Lambda \oplus \Lambda$ is such that $\alpha x=\beta p$. This element belongs to $\Lambda x \cap \wedge p=I(x, b) p$, and it follows that $\alpha=\beta p x^{-1}$. If $\left(a: a^{\prime}\right)=1$, then $(p: b)=1$ and there exist $s, t \in R$ such that $1=s p+t b$. We define the map $\psi: \Lambda \oplus \Lambda \rightarrow \Lambda$ by $\psi(\alpha, \beta)=\alpha x s+\beta$ bt. It is easy to verify that $\operatorname{im} \psi \subseteq \mathrm{I}(x, b)$ and that $\psi \circ \gamma=\operatorname{Id}_{I(x, b)}$. As a consequence, the sequence (1) splits and $I(x, p)$ is a projective $\Lambda$-module.

In particular, for the algebras introduced in Section 2 we have the following:

Corollary 3.4. For all $\mathrm{q} \in \mathrm{k} \backslash\{0,1\}$ and all $\mathrm{a} \in \mathrm{k}[\mathrm{h}]$ we have

$$
\operatorname{gldim} \Lambda\left(k[h], \sigma_{q}, a\right)<\infty \Longrightarrow \operatorname{gldim} \Lambda\left(k[h], \sigma_{q}, a\right)=2 \text {. }
$$

Proof. It follows from [4, Thm. 2.7] that if the global dimension of $\Lambda(R, \sigma, a)$ is finite, it equals either gldim $R$ or gldim $R+1$. In the situation of the corollary, then, gldim $\Lambda(k[h], \sigma, a) \in\{1,2\}$ if it is finite. Moreover, using [4, Thm. 3.7], we see that $\operatorname{gldim} \Lambda(k[h], \sigma, a)=2$ if and only if either (i) there is a maximal ideal of $k[\mathrm{~h}]$ of height 1 with finite orbit under $\sigma$, or (ii) if there are maximal ideals $\mathfrak{p}, \mathfrak{q}$ of $k[h]$ of height 1 such that $\sigma^{\mathfrak{i}}(\mathfrak{p})=\mathfrak{q}$ for some $i \neq 0, i \in \mathbb{Z}$ and $a \in \mathfrak{p} \cap \mathfrak{q}$. Since the ideal $(h)$ of $k[h]$ is obviously fixed by $\sigma$ and it is of height 1 , we are always in case (i), and the corollary follows.

The conditions ( $i$ ) and (ii) mentioned in the proof of this corollary are not exclusive. Indeed, most of the complication encountered in the computations that follow arises when the algebra $A$ satisfies condition (ii) or, in other words, when the polynomial a has two roots in the same orbit under $\sigma_{\mathrm{q}}$. 


\section{A projective resolution}

The purpose of this section is to construct a projective resolution of the Bavula algebra A. We do this in two steps, using an algebra $B_{l}$ as an intermediate step, as in [9].

\subsection{Smith algebras}

Fix a polynomial $l=\sum_{i=0}^{m} \lambda_{i} H^{i} \in k[H]$, with $m>0$ and $\lambda_{m} \neq 0$. We consider the k-algebra $B_{l}$, or simply $B$, with generators $Y, H$ and $X$ subject to the relations

$$
\mathrm{HY}=\mathrm{qYH}, \quad[X, \mathrm{Y}]=\mathrm{l}, \quad \mathrm{XH}=\mathrm{qHX} .
$$

This algebra was considered by P. Smith in [16], observing that it is in many aspects similar to the enveloping algebra $U\left(\mathfrak{s l}_{2}\right)$; we will call it a Smith algebra.

The set $\left\{Y^{i} H^{j} X^{k}: i, j, k \geq 0\right\}$ is a basis of $B$ as a k-module. Let $V=k Y \oplus k H \oplus k X \subset B$. Setting $|X|=|Y|=1$ and $|\mathrm{H}|=0$ we obtain a grading on $T V$, which induces an increasing filtration on $B$; let us write $\bar{Y}, \bar{H}$ and $\bar{X}$ for the principal symbols of $Y, H$ and $X$, respectively, in $\bar{B}=\operatorname{gr} B$. Then $\bar{B}$ is the k-algebra generated by $\bar{Y}, \bar{H}$ and $\bar{X}$, subject to the relations

$$
\overline{\mathrm{HY}}=\mathrm{q} \overline{\mathrm{YH}}, \quad[\overline{\mathrm{X}}, \overline{\mathrm{Y}}]=0, \quad \overline{\mathrm{XH}}=\mathrm{q} \overline{\mathrm{HX}} .
$$

Of course, $\bar{V} \cong g r V$ is spanned by $\bar{X}, \bar{Y}$ and $\bar{H}$, and these elements are $k$-linearly independent.

We will use frequently the following notation: given a function $f$ of two integer arguments, and $i \in \mathbb{N}_{0}$, we will write

$$
\int_{i} f(s, t)=\sum_{\substack{s+t+1=i \\ 0 \leq s, t}} f(s, t) .
$$

In particular, in such an "integral" expression, the indices $s$ and $t$ are not free. We note that the identity

$$
\int_{i} f(s+1, t)-\int_{i} f(s, t+1)=f(i, 0)-f(0, i)
$$

holds for all $f$ and $i$ : we will make use of it repeatedly.

Consider now the complex of $\mathrm{B}^{\mathrm{e}}$-projective modules over $\mathrm{B}$

$$
0 \longrightarrow \mathrm{B}\left|\wedge^{3} \mathrm{~V}\right| \mathrm{B} \longrightarrow \mathrm{B}\left|\wedge^{2} \mathrm{~V}\right| \mathrm{B} \stackrel{\mathrm{d}}{\longrightarrow} \mathrm{B}|\mathrm{V}| \mathrm{B} \stackrel{\mathrm{d}}{\longrightarrow} \mathrm{B} \mid \mathrm{B} \stackrel{\mu}{\longrightarrow} \mathrm{B}
$$

with differentials given by

$$
\begin{aligned}
& \mathrm{d}(1|v| 1)=1|v-v| 1, \quad \forall v \in \mathrm{V} ; \\
& \mathrm{d}(1|\mathrm{H} \wedge \mathrm{X}| 1)=1|\mathrm{X}| \mathrm{H}-\mathrm{qH}|\mathrm{X}| 1-\mathrm{q}|\mathrm{H}| \mathrm{X}+\mathrm{X}|\mathrm{H}| 1 ; \\
& \mathrm{d}(1|\mathrm{Y} \wedge \mathrm{X}| 1)=1|\mathrm{X}| \mathrm{Y}-\mathrm{Y}|\mathrm{X}| 1-1|\mathrm{Y}| \mathrm{X}+\mathrm{X}|\mathrm{Y}| 1-\sum_{i} \int_{i} \lambda_{i} \mathrm{H}^{\mathrm{s}}|\mathrm{H}| \mathrm{H}^{\mathrm{t}} \\
& \mathrm{d}(1|\mathrm{Y} \wedge \mathrm{H}| 1)=1|\mathrm{H}| \mathrm{Y}-\mathrm{q} Y|\mathrm{H}| 1-\mathrm{q}|\mathrm{Y}| \mathrm{H}+\mathrm{H}|\mathrm{Y}| 1 ;
\end{aligned}
$$




$$
\begin{aligned}
\mathrm{d}(1|\mathrm{Y} \wedge \mathrm{H} \wedge \mathrm{X}| 1)=1|\mathrm{H} \wedge \mathrm{X}| \mathrm{Y}-\mathrm{q} Y|\mathrm{H} \wedge \mathrm{X}| 1-\mathrm{q}|\mathrm{Y} \wedge \mathrm{X}| \mathrm{H}+\mathrm{qH}|\mathrm{Y} \wedge \mathrm{X}| 1 \\
+\mathrm{q}|\mathrm{Y} \wedge \mathrm{H}| \mathrm{X}-\mathrm{X}|\mathrm{Y} \wedge \mathrm{H}| 1 .
\end{aligned}
$$

The verification that $d^{2}=0$ is a routine computation.

The filtrations on $B$ and on $V$ determine a filtration on the complex (2), whose associated graded complex is

$$
0 \longrightarrow \overline{\mathrm{B}}\left|\wedge^{3} \overline{\mathrm{V}}\right| \overline{\mathrm{B}} \stackrel{\mathrm{d}}{\longrightarrow} \overline{\mathrm{B}}\left|\wedge^{2} \overline{\mathrm{V}}\right| \overline{\mathrm{B}} \stackrel{\mathrm{d}}{\longrightarrow} \overline{\mathrm{B}}|\overline{\mathrm{V}}| \overline{\mathrm{B}} \stackrel{\mathrm{d}}{\longrightarrow} \overline{\mathrm{B}} \mid \overline{\mathrm{B}} \stackrel{\mu}{\longrightarrow} \overline{\mathrm{B}}
$$

with $\overline{\mathrm{B}}^{e}$-linear differentials determined by the conditions

$$
\begin{aligned}
& \mathrm{d}(1|v| 1)=1|v-v| 1, \quad \forall v \in \overline{\mathrm{V}} ; \\
& \mathrm{d}(1|\overline{\mathrm{H}} \wedge \overline{\mathrm{X}}| 1)=1|\overline{\mathrm{X}}| \overline{\mathrm{H}}-\mathrm{q} \overline{\mathrm{H}}|\overline{\mathrm{X}}| 1-\mathrm{q}|\overline{\mathrm{H}}| \overline{\mathrm{X}}+\overline{\mathrm{X}}|\overline{\mathrm{H}}| 1 ; \\
& \mathrm{d}(1|\overline{\mathrm{Y}} \wedge \overline{\mathrm{X}}| 1)=1|\overline{\mathrm{X}}| \overline{\mathrm{Y}}-\overline{\mathrm{Y}}|\overline{\mathrm{X}}| 1-1|\overline{\mathrm{Y}}| \overline{\mathrm{X}}+\overline{\mathrm{X}}|\overline{\mathrm{Y}}| 1 ; \\
& \mathrm{d}(1|\overline{\mathrm{Y}} \wedge \overline{\mathrm{H}}| 1)=1|\overline{\mathrm{H}}| \overline{\mathrm{Y}}-\mathrm{q} \overline{\mathrm{Y}}|\overline{\mathrm{H}}| 1-\mathrm{q}|\overline{\mathrm{Y}}| \overline{\mathrm{H}}+\overline{\mathrm{H}}|\overline{\mathrm{Y}}| 1 ; \\
& \mathrm{d}(1|\overline{\mathrm{Y}} \wedge \overline{\mathrm{H}} \wedge \overline{\mathrm{X}}| 1)=1|\overline{\mathrm{H}} \wedge \overline{\mathrm{X}}| \overline{\mathrm{Y}}-\mathrm{q} \overline{\mathrm{Y}}|\overline{\mathrm{H}} \wedge \overline{\mathrm{X}}| 1-\mathrm{q}|\overline{\mathrm{Y}} \wedge \overline{\mathrm{X}}| \overline{\mathrm{H}}+\mathrm{q} \overline{\mathrm{H}}|\overline{\mathrm{Y}} \wedge \overline{\mathrm{X}}| 1 \quad+\mathrm{q}|\overline{\mathrm{Y}} \wedge \overline{\mathrm{H}}| \overline{\mathrm{X}}-\overline{\mathrm{X}}|\overline{\mathrm{Y}} \wedge \overline{\mathrm{H}}| 1 .
\end{aligned}
$$

This complex is exact. Indeed, there is a left $\overline{\mathrm{B}}$-linear contraction given by

$$
\begin{aligned}
& s(1)=1 \mid 1 ; \\
& s\left(1 \mid \bar{Y}^{i} \bar{H}^{j} \bar{X}^{k}\right)=\sum_{i} \int_{i} \bar{Y}^{s}|\bar{Y}| \bar{Y}^{t} \bar{H}^{j} \bar{X}^{k}+\sum_{i} \int_{j} \bar{Y}^{i} \bar{H}^{s}|\bar{H}| \bar{H}^{t} \bar{X}^{k}+\sum_{i} \int_{k} \bar{Y}^{i} \bar{H}^{j} \bar{X}^{s}|\bar{X}| \bar{X}^{t} ; \\
& s\left(1|\bar{Y}| \bar{Y}^{i} \bar{H}^{j} \bar{X}^{k}\right)=0 \\
& s\left(1|\bar{H}| \bar{Y}^{i} \bar{H}^{j} \bar{X}^{k}\right)=\sum_{i} \int_{i} q^{s} \bar{Y}^{s}|\bar{Y} \wedge \bar{H}| \bar{Y}^{t} \bar{H}^{j} \bar{X}^{k} ; \\
& s\left(1|\bar{X}| \bar{Y}^{i} \bar{H}^{j} \bar{X}^{k}\right)=\sum_{i} \int_{i} \bar{Y}^{s}|\bar{Y} \wedge \bar{X}| \bar{Y}^{t} \bar{H}^{j} \bar{X}^{k}+\sum_{i} \int_{j} q^{s} \bar{Y}^{i} \bar{H}^{s}|\bar{H} \wedge \bar{X}| \bar{H}^{t} \bar{X}^{k} ; \\
& s\left(1|\bar{H} \wedge \bar{X}| \bar{Y}^{i} \bar{H}^{j} \bar{X}^{k}\right)=\sum_{i} \int_{i} q^{s} \bar{Y}^{s}|\bar{Y} \wedge \bar{H} \wedge \bar{X}| \bar{Y}^{t} \bar{H}^{j} \bar{X}^{k} ; \\
& s\left(1|\bar{Y} \wedge \bar{X}| \bar{Y}^{i} \bar{H}^{j} \bar{X}^{k}\right)=0 \\
& s\left(1|\bar{Y} \wedge \bar{H}| \bar{Y}^{i} \bar{H}^{j} \bar{X}^{k}\right)=0
\end{aligned}
$$

It follows that the complex (2) is a $\mathrm{B}^{\mathrm{e}}$-projective resolution of $\mathrm{B}$.

\subsection{Bavula algebras}

Next we construct a resolution of our Bavula algebra as a bimodule over itself. Let $l=\sigma(a)-a$; then $\operatorname{deg} a \geq \operatorname{deg} l$ and $l=\sum_{i=0}^{N} \lambda_{i} h^{i}$ with $\lambda_{i}=\left(q^{i}-1\right) \alpha_{i}$. We consider the Smith algebra $B=B_{l}$ corresponding to the polynomial $l$, and the element $\Omega=Y X-a \in B$. A simple computation shows that $\Omega=X Y-\sigma(a)$ and that $\Omega$ is central in $B$. In particular, $B \Omega=\Omega B$ is a two-sided ideal of $B$ and the quotient $B / \Omega B$ is isomorphic to $A$ via an isomorphism which sends the classes of $Y, H$ and $X$ to $y, h$ and $x$ respectively. We will identify $A$ with the quotient. 
Let $\pi: \mathrm{B} \rightarrow \mathrm{A}$ denote the canonical projection. Since $\Omega$ is not a zero divisor in $\mathrm{B}$, the complex

$$
0 \longrightarrow \mathrm{B} \stackrel{\Omega}{\longrightarrow} \mathrm{B} \stackrel{\pi}{\longrightarrow} \mathrm{A} \longrightarrow 0
$$

is a projective resolution of $A$ as a B-module both on the left and on the right; here the first arrow is simply the multiplication by $\Omega$. On the other hand, by applying the functor $(-) \otimes_{\mathrm{B}} \mathrm{A}$ to the resolution (2) of $\mathrm{B}$ as $\mathrm{B}^{e}$-module given in the previous subsection, we obtain the complex

$$
0 \longrightarrow \mathrm{B}\left|\wedge^{3} \mathrm{~V}\right| \mathrm{A} \stackrel{\mathrm{d}}{\longrightarrow} \mathrm{B}\left|\wedge^{2} \mathrm{~V}\right| \mathrm{A} \stackrel{\mathrm{d}}{\longrightarrow} \mathrm{B}|\mathrm{V}| \mathrm{A} \stackrel{\mathrm{d}}{\longrightarrow} \mathrm{B} \mid \mathrm{A} \stackrel{\mu}{\longrightarrow} \mathrm{A} \longrightarrow 0
$$

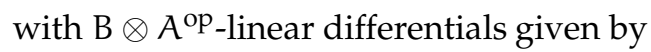

$$
\begin{aligned}
& \mathrm{d}(1|v| 1)=1|v-v| 1, \quad \forall v \in \mathrm{V} ; \\
& \mathrm{d}(1|\mathrm{H} \wedge \mathrm{X}| 1)=1|\mathrm{X}| \mathrm{h}-\mathrm{qH}|\mathrm{X}| 1-\mathrm{q}|\mathrm{H}| \mathrm{x}+\mathrm{X}|\mathrm{H}| 1 ; \\
& \mathrm{d}(1|\mathrm{Y} \wedge \mathrm{X}| 1)=1|\mathrm{X}| \mathrm{y}-\mathrm{Y}|\mathrm{X}| 1-1|\mathrm{Y}| \mathrm{x}+\mathrm{X}|\mathrm{Y}| 1-\sum_{\mathrm{i}} \int_{\mathrm{i}} \lambda_{\mathrm{i}} \mathrm{H}^{\mathrm{s}}|\mathrm{H}| \mathrm{h}^{\mathrm{t}} ; \\
& \mathrm{d}(1|\mathrm{Y} \wedge \mathrm{H}| 1)=1|\mathrm{H}| \mathrm{y}-\mathrm{q} \mathrm{Y}|\mathrm{H}| 1-\mathrm{q}|\mathrm{Y}| \mathrm{h}+\mathrm{H}|\mathrm{Y}| 1 ; \\
& \mathrm{d}(1|\mathrm{Y} \wedge \mathrm{H} \wedge \mathrm{X}| 1)=1|\mathrm{H} \wedge \mathrm{X}| \mathrm{y}-\mathrm{q} \mathrm{Y}|\mathrm{H} \wedge \mathrm{X}| 1-\mathrm{q}|\mathrm{Y} \wedge \mathrm{X}| \mathrm{h}+\mathrm{qH}|\mathrm{Y} \wedge \mathrm{X}| 1+\mathrm{q}|\mathrm{Y} \wedge \mathrm{H}| \mathrm{x}-\mathrm{X}|\mathrm{Y} \wedge \mathrm{H}| 1 .
\end{aligned}
$$

The homology of this complex is $\operatorname{Tor}_{\bullet}^{B}(B, A)$, so that it is in fact acyclic. This means that (4) is a projective resolution of $A$ as a left $B$-module.

There exist morphisms between the two resolutions (3) and (4) of the left B-module A lifting the identity map of A:

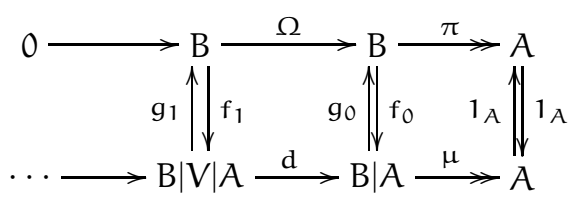

given by

$$
\begin{array}{ll}
f_{0}(1)=1 \mid 1 ; & f_{1}(1)=-Y|X| 1-1|Y| x+\sum_{i} \int_{i} \alpha_{i} H^{s}|H| h^{t} \\
g_{0}\left(1 \mid y^{i} h^{j}\right)=Y^{i} H^{j} ; & g_{0}\left(1 \mid h^{j} \chi^{k}\right)=H^{j} X^{k} \\
g_{1}\left(1|Y| y^{i} h^{j}\right)=0 ; & g_{1}\left(1|Y| h^{j} x^{k+1}\right)=-q^{-j} H^{j} X^{k} \\
g_{1}\left(1|H| y^{i} h^{j}\right)=0 ; & g_{1}\left(1|H| h^{j} x^{k}\right)=0 ; \\
g_{1}\left(1|X| y^{i+1} h^{j}\right)=-Y^{i} H^{j} ; & g_{1}\left(1|X| h^{j} x^{k}\right)=0 .
\end{array}
$$

Using (3), the computation of $\operatorname{Tor}_{\bullet}^{B}(A, A)$ is immediate because the only relevant differential vanishes, and we see that

$$
\operatorname{Tor}_{\mathfrak{p}}^{B}(A, A)= \begin{cases}A \otimes_{B} B, & p=0 \\ A \otimes_{B} B, & p=1 \\ 0, & p \geq 2\end{cases}
$$


Since $\operatorname{Tor}_{\bullet}^{B}(A, A)$ can be calculated from any resolution of $A$ as left $B$-module, the complex obtained by applying the functor $A \otimes_{B}(-)$ to the resolution (4), that is

$$
0 \longrightarrow A\left|\wedge^{3} \mathrm{~V}\right| \mathrm{A} \stackrel{\mathrm{d}}{\longrightarrow} \mathrm{A}\left|\wedge^{2} \mathrm{~V}\right| \mathrm{A} \stackrel{\mathrm{d}}{\longrightarrow} \mathrm{A}|\mathrm{V}| \mathrm{A} \stackrel{\mathrm{d}}{\longrightarrow} \mathrm{A} \mid \mathrm{A}
$$

with $A^{e}$-linear differential:

$$
\begin{aligned}
& \mathrm{d}(1|v| 1)=1|\pi(v)-\pi(v)| 1, \quad \forall v \in \mathrm{V} ; \\
& \mathrm{d}(1|\mathrm{H} \wedge \mathrm{X}| 1)=1|\mathrm{X}| \mathrm{h}-\mathrm{qh}|\mathrm{X}| 1-\mathrm{q}|\mathrm{H}| x+x|\mathrm{H}| 1 ; \\
& \mathrm{d}(1|\mathrm{Y} \wedge \mathrm{X}| 1)=1|X| y-y|X| 1-1|\mathrm{Y}| x+x|\mathrm{Y}| 1-\sum_{\mathrm{i}} \int_{\mathrm{i}} \lambda_{\mathrm{i}} \mathrm{h}^{\mathrm{s}}|\mathrm{H}| \mathrm{h}^{\mathrm{t}} ; \\
& \mathrm{d}(1|\mathrm{Y} \wedge \mathrm{H}| 1)=1|\mathrm{H}| \mathrm{y}-\mathrm{q} y|\mathrm{H}| 1-\mathrm{q}|\mathrm{Y}| \mathrm{h}+\mathrm{h}|\mathrm{Y}| 1 ; \\
& \mathrm{d}(1|\mathrm{Y} \wedge \mathrm{H} \wedge \mathrm{X}| 1)=1|\mathrm{H} \wedge \mathrm{X}| \mathrm{y}-\mathrm{q}|\mathrm{H}| \mathrm{H} \wedge \mathrm{X}|1-\mathrm{q}| \mathrm{Y} \wedge \mathrm{X}|\mathrm{h}+\mathrm{qh}| \mathrm{Y} \wedge \mathrm{X}|1+\mathrm{q}| \mathrm{Y} \wedge \mathrm{H}|x-\mathrm{x}| \mathrm{Y} \wedge \mathrm{H} \mid 1,
\end{aligned}
$$

has homology isomorphic to $\operatorname{Tor}_{\bullet}^{B}(A, A)$. Using the morphisms $f_{\bullet}$ and $g_{\bullet}$ from (5), we see that the homology of the complex (7) is freely generated as left $A$-module by the classes of the cycles $1 \mid 1 \in A \otimes A$ and

$$
y|X| 1+1|Y| x-\sum_{i} \int_{i} \alpha_{i} h^{s}|H| h^{t} \in A \otimes V \otimes A,
$$

of degrees 0 and 1, respectively.

\subsection{The resolution}

Next we consider the third-quadrant double complex $X_{\bullet}, \bullet$ depicted in the following diagram

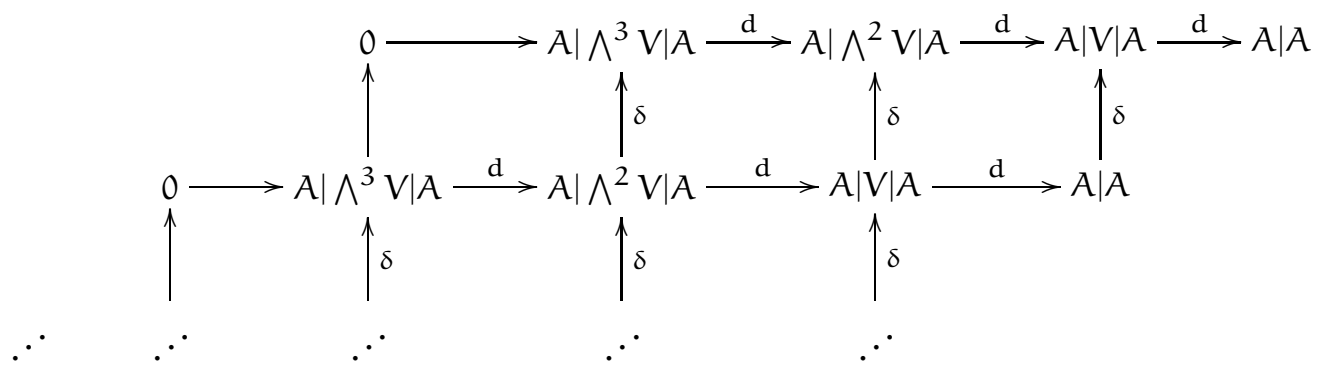

so that $X_{p, q}=A\left|\wedge^{p-q} V\right| A$ if $q \geq 0$ and $X_{p, q}=0$ otherwise, with horizontal $A^{e}$-linear differentials $d$, of bidegree $(-1,0)$, given as in $(7)$, and vertical differentials $\delta$, of bidegree $(0,1)$, given by

$$
\begin{aligned}
& \delta(1 \mid 1)=y|X| 1+1|Y| x-\sum_{i} \int_{i} \alpha_{i} h^{s}|H| h^{t} \\
& \delta(1|Y| 1)=-y|Y \wedge X| 1+\sum_{i} \int_{i} \alpha_{i} q^{t} h^{s}|Y \wedge H| h^{t} \\
& \delta(1|H| 1)=1|Y \wedge H| x-y|H \wedge X| 1 ; \\
& \delta(1|X| 1)=1|Y \wedge X| x-\sum_{i} \int_{i} \alpha_{i} q^{s} h^{s}|H \wedge X| h^{t} ; \\
& \delta(1|Y \wedge H| 1)=y|Y \wedge H \wedge X| 1 ;
\end{aligned}
$$




$$
\begin{aligned}
& \delta(1|\mathrm{Y} \wedge \mathrm{X}| 1)=\sum_{i} \int_{i} \alpha_{i} q^{i-1} h^{s}|\mathrm{Y} \wedge \mathrm{H} \wedge X| \mathrm{h}^{\mathrm{t}} \\
& \delta(1|\mathrm{H} \wedge \mathrm{X}| 1)=1|\mathrm{Y} \wedge \mathrm{H} \wedge \mathrm{X}| \mathrm{x} .
\end{aligned}
$$

A direct computation shows that it is indeed a complex with anti-commuting differentials.

To compute the homology of the total complex $\operatorname{Tot} X_{\bullet, \bullet}$ we use the spectral sequence $E$ which arises from the filtration by rows. The differential on the first page $E^{0}$ of this spectral sequence is the horizontal differential $\mathrm{d}$ on $\mathrm{X}_{\bullet}, \bullet$, , and we have essentially computed the corresponding homology in (6): we see from this that the second page $E^{1}$ of $E$ is, up to isomorphism, as in the following

\begin{tabular}{|c|c|c|c|c|}
\hline & & 0 & 0 & $A$ \\
\hline & 0 & 0 & $\begin{array}{l}A \\
\uparrow_{\mathrm{d}^{1}}\end{array}$ & $A$ \\
\hline 0 & 0 & $A$ & A & \\
\hline
\end{tabular}
diagram:

Consequently, the only components of the differential $\mathrm{d}^{1}$ which can possibly be non zero are the maps $d_{p, p}^{1}: E_{p, p}^{1} \rightarrow E_{p, p-1}^{1}$, with $p \geq 1$, and they are induced by the vertical differentials $\delta$ in $X_{\bullet}, \bullet$. We know that $E_{p, p}^{1, p}$ and $E_{p, p-1}^{1}$ are free left $A$-modules on the horizontal homology classes of $1 \mid 1 \in X_{p, p}$ and $\omega=y|X| 1+1|Y| x-\sum_{i} \int_{i} \alpha_{i} h^{s}|H| h^{t} \in X_{p, p-1}$, respectively. In view of the definition of $\delta, d^{1}([1 \mid 1])=[\omega]$, and, since $d^{1}$ is A-linear, this shows that all components of $d^{1}$ which are not trivially zero are isomorphisms.

It follows that the complex $\operatorname{Tot} X_{\bullet}, \bullet$ is acyclic over $A$, with augmentation given by the multiplication map $\mu: X_{0,0}=A \otimes A \rightarrow A$ and, since its components are free $A^{e}$-modules, it is in fact a projective resolution of $A$ as $A^{e}$-module.

We consider the grading $V$ such that $Y, H$ and $X$ are homogeneous of degrees 1,0 and -1 , respectively. This, together with the grading of $A$ by weights, induces a grading on the complex $X_{\bullet}, \bullet$ such the differentials are homogeneous. It follows that the complexes obtained by applying the functors $A \otimes_{A^{e}}(-)$ and hom $A^{e}(-, A)$ below will also be graded by weights in a natural way.

\section{Hochschild homology}

In this section we will compute the Hochschild homology of $A$ using the resolution described in the previous section and a spectral sequence argument.

Applying the functor $A \otimes_{A^{e}}$ - to $X_{\bullet}, \bullet$ and identifying $A \otimes_{A^{e}}\left(A \otimes \wedge^{p} \vee \otimes A\right)$ with $A \otimes \wedge^{p} V$ in the natural way, we get a double complex such that the homology of its total complex is $\operatorname{HH}_{*}(A)$, 
the Hochschild homology of $A$ with coefficients in itself. This double complex is

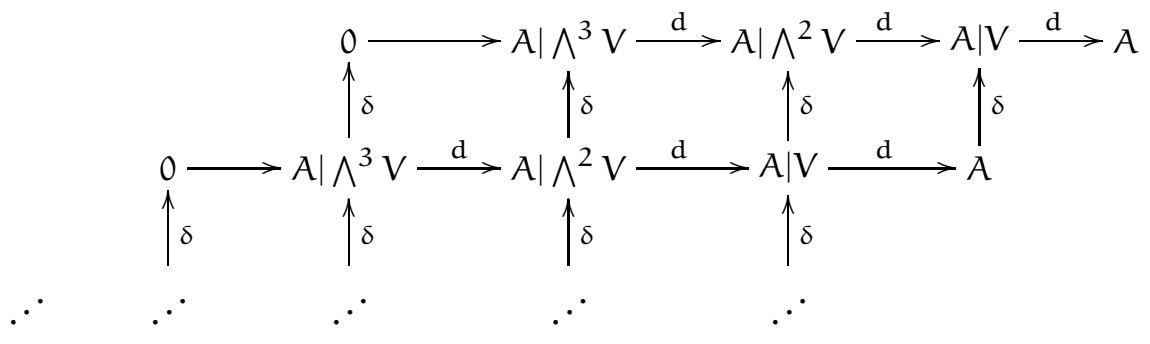

with differentials given by

$$
\begin{aligned}
& d(u \mid Y)=[y, u], \\
& d(u \mid H)=[h, u], \\
& d(u \mid X)=[x, u], \\
& d(u \mid Y \wedge H)=[y, u]_{q}\left|H+[u, h]_{q}\right| Y, \\
& d(u \mid Y \wedge X)=[y, u]^{X}+[u, x]\left|Y-\sum_{i} \lambda_{i} \int_{i} h^{t} u h^{s}\right| H, \\
& d(u \mid H \wedge X)=[h, u]_{q}\left|X+[u, x]_{q}\right| H, \\
& d(u \mid Y \wedge H \wedge X)=[y, u]_{q}|H \wedge X+q[u, h]| Y \wedge X-[u, x]_{q} \mid Y \wedge H,
\end{aligned}
$$

and

$$
\begin{aligned}
& \delta(u)=u y|X+x u| Y-\sum_{i} \alpha_{i} \int_{i} h^{t} u h^{s} \mid H, \\
& \delta(u \mid Y)=-u y\left|Y \wedge X+\sum_{i} \alpha_{i} \int_{i} q^{t} h^{t} u h^{s}\right| Y \wedge H, \\
& \delta(u \mid H)=x u|Y \wedge H-u y| H \wedge X, \\
& \delta(u \mid X)=x u\left|Y \wedge X-\sum_{i} \alpha_{i} \int_{i} q^{s} h^{t} u h^{s}\right| H \wedge X, \\
& \delta(u \mid Y \wedge H)=u y \mid Y \wedge H \wedge X, \\
& \delta(u \mid Y \wedge X)=\sum_{i} \alpha_{i} \int_{i} q^{i-1} h^{t} u h^{s} \mid Y \wedge H \wedge X, \\
& \delta(u \mid H \wedge X)=x u \mid Y \wedge H \wedge X .
\end{aligned}
$$

We will use the filtration by columns on this complex and denote $E$ the corresponding spectral sequence, which, as the complex $A \otimes_{A^{e}} X_{\bullet}, \bullet$ itself, is graded by weights. We are going to write $\mathrm{HH}_{\bullet}(A)^{(r)}$ and $E^{(r)}$ the components of weight $r$ in $H_{\bullet}(A)=H\left(A \otimes_{A^{e}} X_{\bullet}, \bullet\right)$ and $E$.

\subsection{First Page}

Let $X$ be the complex

$$
0 \longrightarrow \mathrm{A} \stackrel{\delta}{\longrightarrow} \mathrm{A}|\mathrm{V} \stackrel{\delta}{\longrightarrow} \mathrm{A}| \wedge^{2} \mathrm{~V} \stackrel{\delta}{\longrightarrow} \mathrm{A} \mid \wedge^{3} \mathrm{~V} \longrightarrow 0
$$

graded so that $A$ and $A \mid \Lambda^{3} V$ are in degrees 0 and 3, respectively, and with differentials as in (9a)(9g). It is clear that $E_{p, q}^{1}=H_{p-q}(X)$ for all $q>0$ and that the $E_{p, 0}^{1}$ can be seen as cokernels of the differentials of $X$. 
For each $r \in \mathbb{Z}$, let $\mathcal{X}^{(r)}$ be the homogeneous component of weight $r$. In this subsection, we compute $\mathrm{H}_{\bullet}(X)=\bigoplus_{\mathrm{r} \in \mathbb{Z}} \mathrm{H}_{\bullet}\left(X^{(\mathrm{r})}\right)$.

Proposition 5.1. If $r \in \mathbb{Z}$ is non zero, then the complex $X^{(r)}$ is exact. On the other hand, there are isomorphisms of S-modules

$$
H_{p}\left(X^{(0)}\right) \cong \begin{cases}k[h] /(c), & \text { if } 2 \leq p \leq 3 \\ 0, & \text { otherwise. }\end{cases}
$$

Proof. One way to organize the computation is as follows:

- If $u=p \in X_{0}^{(0)}$, with $p \in k[h]$, then

$$
\delta(u)=y \sigma(p)|X+\sigma(p) x| Y-a^{\prime} p \mid H .
$$

As $A$ is a domain, it follows immediately that $\delta$ is a monomorphism and that $\mathrm{H}_{0}\left(X^{(0)}\right)=0$. - Let $u=p_{1} x\left|Y+p_{2}\right| H+y p_{3} \mid X \in X_{1}^{(0)}$, with $p_{1}, p_{2}, p_{3} \in k[h]$. We know that

$$
\delta(u)=\left(p_{1} \sigma\left(a^{\prime}\right)+\sigma\left(p_{2}\right)\right) x\left|Y \wedge H+\sigma(a)\left(p_{3}-p_{1}\right)\right| Y \wedge X-y\left(p_{3} \sigma\left(a^{\prime}\right)+\sigma\left(p_{2}\right)\right) \mid H \wedge X .
$$

Since $A$ is a domain, we see that $\delta(u)=0$ if and only if $p_{1}=p_{3}$ and $p_{2}=-\sigma^{-1}\left(p_{1}\right) a^{\prime}$. This description of cyles together with the expression (11) of boundaries imply that $\mathrm{H}_{1}\left(X^{(0)}\right)=0$.

- Let $u=p_{1} x\left|Y \wedge H+p_{2}\right| Y \wedge X+y p_{3} \mid H \wedge X \in X_{2}^{(0)}$. A computation shows that

$$
\delta(u)=\left(p_{1} \sigma(a)+p_{2} \sigma\left(a^{\prime}\right)+\sigma(a) p_{3}\right) \mid Y \wedge H \wedge X .
$$

Suppose that $u \in \operatorname{ker} \delta$, so $p_{1} \sigma(a)+p_{2} \sigma\left(a^{\prime}\right)+\sigma(a) p_{3}=0$. It follows immediately from this that $\sigma\left(\frac{a}{c}\right)\left(p_{1}+p_{3}\right)=-\sigma\left(\frac{a^{\prime}}{c}\right) p_{2}$. Since $a / c$ and $a^{\prime} / c$ are coprime, there exists $g \in k[h]$ such that $p_{1}+p_{3}=-\sigma\left(\frac{a^{\prime}}{c}\right) g$ and $p_{2}=\sigma\left(\frac{a}{c}\right) g$. If $v, r \in k[h]$ are such that $g=v \sigma(c)+r$ and $\operatorname{deg} r<\operatorname{deg} c$, then $u$ is homologous to

$$
u-\delta\left(\sigma^{-1}\left(p_{1}\right)|H+y v| X\right)=\operatorname{rr}\left(\frac{a}{c}\right)\left|Y \wedge X-y r \sigma\left(\frac{a^{\prime}}{c}\right)\right| H \wedge X .
$$

It follows from this that every homology class of degree 2 in $X^{(0)}$ is represented by a cycle of the form $r \sigma\left(\frac{a}{c}\right)\left|Y \wedge X-\operatorname{yr} \sigma\left(\frac{a^{\prime}}{c}\right)\right| H \wedge X$ with $r \in k[h]$ with $\operatorname{deg} r<\operatorname{deg} c=M$. In view of the formula (12), one of these cycles is a boundary if and only if it is zero, and we can then conclude that $\mathrm{H}_{2}\left(X^{(0)}\right) \cong k[h] /(\sigma(c)) \cong k[h] /(c)$.

- It follows immediately from (13) that $\delta\left(X_{2}^{(0)}\right)=\sigma(c) k[h] \mid Y \wedge H \wedge X$, so $H_{3}\left(X^{(0)}\right) \cong k[h] /(c)$.

We fix now $r>0$, and show that $\mathcal{X}^{(r)}$ is exact.

- Let $u \in X_{0}^{(r)}$, so that $u=y^{r} p$ for some $p \in k[h]$. Then

$$
\delta(u)=y^{r-1} \sigma^{r}(a) p\left|Y-y^{r} p \sum_{i} \alpha_{i}[i] q^{r} h^{i-1}\right| H+y^{r+1} \sigma(p) \mid X,
$$

and we see immediately that this is zero if and only if $p=0$, so $\mathrm{H}_{0}\left(X^{(r)}\right)=0$. 
- Let $u=y^{r-1} p_{1}\left|Y+y^{r} p_{2}\right| H+y^{r+1} p_{3} \mid X \in X_{1}^{(r)}$ with $p_{1}, p_{2}, p_{3} \in k[h]$. As

$$
\begin{array}{r}
\delta(u)=y^{r-1}\left(p_{1} \sum_{i} \alpha_{i}[i]_{q^{r}} h^{i-1}+\sigma^{r}(a) p_{2}\right)\left|Y \wedge H+y^{r}\left(-\sigma\left(p_{1}\right)+\sigma^{r+1}(a) p_{3}\right)\right| Y \wedge X \\
\left.-y^{r+1}\left(\sigma\left(p_{2}\right)+p_{3} \sum_{i} \alpha_{i} q^{i-1}[i]\right]_{q} h^{i-1}\right) \mid H \wedge X,
\end{array}
$$

we have that $u$ is a cycle if and only if

$$
\begin{aligned}
& p_{1} \sum_{i} \alpha_{i}[i]_{q^{r}} h^{i-1}+\sigma^{r}(a) p_{2}=0, \\
& \sigma^{r+1}(a) p_{3}=\sigma\left(p_{1}\right), \\
& \sigma\left(p_{2}\right)+p_{3} \sum_{i} \alpha_{i} q^{i-1}[i]_{q^{r}} h^{i-1}=0 .
\end{aligned}
$$

The first one follows from the other two, so we can drop it, and we can replace the remaining ones by

$$
\begin{aligned}
& p_{2}=-\sigma^{-1}\left(p_{3}\right) \sum_{i} \alpha_{i}[i]_{q^{r}} h^{i-1}, \\
& p_{1}=\sigma^{r}(a) \sigma^{-1}\left(p_{3}\right) .
\end{aligned}
$$

We thus obtain a description of all 1-cycles in $X^{(r)}$ and comparing it with (14), we see that they are all boundaries: it follows that $\mathrm{H}_{1}\left(X^{(\mathrm{r})}\right)=0$.

- For $u=y^{r-1} p_{1}\left|Y \wedge H+y^{r} p_{2}\right| Y \wedge X+y^{r+1} p_{3} \mid H \wedge X \in X_{2}^{(r)}$ with $p_{1}, p_{2}, p_{3} \in k[h]$, we have

$$
\delta(u)=y^{r}\left(\sigma\left(p_{1}\right)+p_{2} \sum_{i} \alpha_{i} q^{i-1}[i]_{q^{r}} h^{i-1}+\sigma^{r+1}(a) p_{3}\right) \mid Y \wedge H \wedge X .
$$

If $u$ is a cycle, then $p_{1}=-\sigma^{-1}\left(p_{2} \sum_{i} \alpha_{i} q^{i-1}[i]_{q^{r}} h^{i-1}+\sigma^{r+1}(a) p_{3}\right)$ so that, in fact,

$$
u=-\delta\left(y^{r-1} \sigma^{-1}\left(p_{2}\right)\left|Y+y^{r} \sigma^{-1}\left(p_{3}\right)\right| H\right) .
$$

It follows from this that $\mathrm{H}_{2}\left(X^{(\mathrm{r})}\right)=0$.

- For each $p \in k[h]$, we have that $\delta\left(y^{r-1} \sigma^{-1}(p) \mid Y \wedge H\right)=y^{r} p \mid Y \wedge H \wedge X$. This means that $\delta\left(X_{2}^{r}\right)=X_{3}^{r}$, so $H_{3}\left(X^{(r)}\right)=0$.

At this point, we know most of the second page of our spectral sequence:

Corollary 5.2. Let $r \in \mathbb{Z}$ a weight. The dimensions of the vector spaces appearing in the homogeneous component of weight $\mathrm{r}$ of $\mathrm{E}^{1}$ are

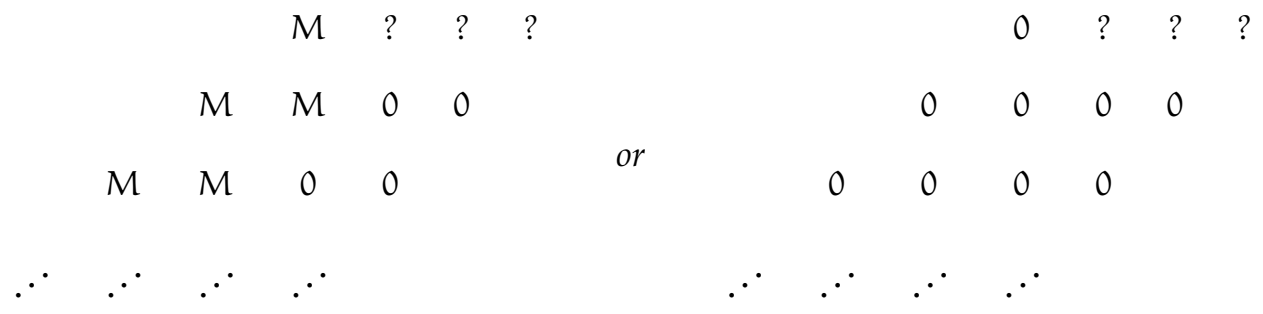

depending on whether $r=0$ or not. The question marks denote vector spaces for which we still do not know the dimension. 


\subsection{Second page}

In view of the shape of $E^{1}$, we have $E^{\infty}=E^{2}$. The following proposition takes care of the latter, except for its first row, and the rest of this section will be devoted to the computation of the few remaining vector spaces.

Proposition 5.3. For each $p \geq 0$, the differential $\mathrm{d}_{\mathrm{p}+3, \mathrm{p}}^{1}: \mathrm{E}_{\mathrm{p}+3, \mathrm{p}}^{1} \rightarrow \mathrm{E}_{\mathrm{p}+2, \mathrm{p}}^{1}$ vanishes. In consequence, except for the vector spaces denoted with question marks in the diagrams of Corollary 5.2, the $\mathrm{E}^{\infty}$ page coincides with the page $\mathrm{E}^{1}$.

Proof. A simple computation shows that if $f \in k[h]$ then

$$
\begin{aligned}
d(f \mid Y \wedge H \wedge X) & =y(1-q \sigma)(f)|H \wedge X-(1-q \sigma)(f) x| Y \wedge H \\
& =\delta\left(\left(q-\sigma^{-1}\right)(f) \mid H\right) .
\end{aligned}
$$

It follows that all the differentials $\mathrm{d}_{\mathrm{p}+3, p}^{2}$ are zero, as claimed, and the computation of $\mathrm{E}^{\infty}$ is immediate except for $\mathrm{E}_{0,0}^{\infty}, \mathrm{E}_{1,0}^{\infty}$ and $\mathrm{E}_{2,0}^{\infty}$.

Corollary 5.4. For all $p \geq 3$ and all $r \in \mathbb{Z}$ there are isomorphisms of S-modules

$$
\mathrm{HH}_{\mathrm{p}}(A)_{(\mathrm{r})} \cong \begin{cases}k[h] /(\mathrm{c}) & \text { if } \mathrm{r}=0 \\ 0 & \text { if } \mathrm{r} \neq 0\end{cases}
$$

Notice that this result is independent of $q$.

Proof. According to the proposition and in view of the shape of the $E^{1}$ page of the spectral sequence, this is a consequence of convergence.

To finish the computation, we need to take care of the spots in the spectral sequence tagged with question marks in the diagrams of Corollary 5.2. We do this in the following two propositions, first for weight zero and then for the remaining ones.

Proposition 5.5. When $\mathrm{q}$ is a root of unity, we have isomorphisms of $\mathcal{S}$-modules

$$
\mathrm{HH}_{\mathrm{p}}(\mathrm{A})^{(0)} \cong \mathrm{E}_{\mathrm{p}, 0}^{2(0)} \cong \begin{cases}\mathrm{k}^{\eta(\mathrm{a})}, & \text { if } \mathrm{p}=0 ; \\ \mathcal{S} \oplus \mathcal{S} \oplus \mathrm{k}^{\eta(c)}, & \text { if } \mathrm{p}=1 ; \\ \mathcal{S} \oplus \mathrm{k}[\mathrm{h}] /(\mathrm{c}), & \text { if } \mathrm{p}=2\end{cases}
$$

with $\eta(f)=N-\frac{1}{e} \operatorname{deg} \mathcal{N}(\mathbf{f})$ for $\mathrm{f} \in \mathrm{k}[\mathrm{h}]$ as in Lemma 2.3. On the other hand, if $\mathrm{q}$ is of infinite order we have isomorphisms

$$
H H_{p}(A)^{(0)} \cong E_{p, 0}^{2(0)} \cong \begin{cases}k^{N}, & \text { if } p=0 \\ k^{M}, & \text { if } p=1 \\ k^{M}, & \text { if } p=2\end{cases}
$$

Proof. We write $\mathrm{E}_{\mathrm{p}, 0}^{1}$ instead of $\mathrm{E}_{\mathrm{p}, 0}^{1(0)}$ throughout this proof, to lighten the notation. 
Homology at $E_{2,0}^{1}$. Suppose $u=p_{1} x\left|Y \wedge H+p_{2}\right| Y \wedge X+y p_{3} \mid H \wedge X \in E_{2,0}^{0}$, with $p_{1}, p_{2}, p_{3} \in k[h]$, lives to $E^{2}$, so that there exists an $f \in k[h]$ such that $d(u)=\delta(f)$. This means that

$$
\begin{aligned}
& (1-\sigma)\left(p_{2}\right)=\sigma(f), \\
& a \sigma^{-1}\left(p_{1}+p_{3}\right)-q \sigma(a)\left(p_{1}+p_{3}\right)-p_{2}\left(q \sigma\left(a^{\prime}\right)-a^{\prime}\right)=-a^{\prime} f .
\end{aligned}
$$

Since $\sigma$ is a automorphism, we can eliminate $f$ obtaining the equivalent equation

$$
a \sigma^{-1}\left(p_{1}+p_{3}\right)-q \sigma(a)\left(p_{1}+p_{3}\right)-p_{2}\left(q \sigma\left(a^{\prime}\right)-a^{\prime}\right)=-a^{\prime} \sigma^{-1}\left((1-\sigma)\left(p_{2}\right)\right),
$$

which we can rewrite more compactly as

$$
(1-q \sigma)\left(a \sigma^{-1}\left(p_{1}+p_{3}\right)+a^{\prime} \sigma^{-1}\left(p_{2}\right)\right)=0 .
$$

It will be necessary to treat two cases separately, since the result depends on whether $q$ is a root of unity or not.

- Suppose first that $\mathrm{q}$ is not a root of 1 . In this case, the map $1-\mathrm{q} \sigma$ is a monomorphism, so (16) is the same as

$$
a \sigma^{-1}\left(p_{1}+p_{3}\right)+a^{\prime} \sigma^{-1}\left(p_{2}\right)=0 .
$$

From this it follows that there exists $g \in k[h]$ such that

$$
\mathrm{p}_{2}=-\sigma\left(\frac{\mathrm{a}}{\mathrm{c}}\right) \mathrm{g}, \quad \mathrm{p}_{1}+\mathrm{p}_{3}=\sigma\left(\frac{\mathrm{a}^{\prime}}{\mathrm{c}}\right) \mathrm{g} .
$$

Let $b, r \in k[h]$ be such that $g=b \sigma(c)+r$ with $\operatorname{deg} r<\operatorname{deg} c$. Then $u$ is homologous to

$$
u+\delta\left(y b\left|X-\sigma^{-1}\left(p_{1}\right)\right| H\right)=\sigma\left(\frac{a}{c}\right) r\left|Y \wedge X+y \sigma\left(\frac{a^{\prime}}{c}\right) r\right| H \wedge X,
$$

and we see that every homology class in $E_{2,0}^{2}$ is represented by a cycle of the form

$$
\sigma\left(\frac{a}{c}\right) r\left|Y \wedge X+y \sigma\left(\frac{a^{\prime}}{c}\right) r\right| H \wedge X
$$

with $r \in k[h]$ with $\operatorname{deg} r<M=\operatorname{deg} c$. Conversely, each element of this form lives to $E^{2}$.

Using (15) we see that the image of $d$ contains the image of $\delta$. On the other hand, the coefficient of $Y \wedge X$ in every non zero element of $\delta\left(X_{1}^{(0)}\right)$ is multiple of $\sigma(a)$, so in particular it has degree at least $\mathrm{N}$ : comparing with (17) we see that $u$ is not in the image of $\delta$. We can therefore conclude that these elements are non zero in $E^{2}$, so that $\operatorname{dim} E_{2,0}^{2}=M$.

- Suppose now that $q$ is a root of 1 . In this case the condition (16) is equivalent to the existence of a singular polynomial $s \in \mathcal{S}$ such that

$$
a \sigma^{-1}\left(p_{1}+p_{3}\right)+a^{\prime} \sigma^{-1}\left(p_{2}\right)=h^{e-1} s .
$$

As $a(0) \neq 0, c$ divides $s$ and it follows from Proposition 2.1(ii) that there exists $s_{1} \in \mathcal{S}$ such that $s=\mathcal{N}(\mathrm{c}) s_{1}$.

Let $\alpha, \beta \in k[h]$ be such that $\frac{a}{c} \alpha+\frac{a^{\prime}}{c} \beta=1$; each solution of the equation (18) is of the form

$$
p_{3}=\sigma\left(h^{e-1} \bar{c} s_{1} \alpha+\frac{a^{\prime}}{c} g\right)-p_{1},
$$




$$
\mathrm{p}_{2}=\sigma\left(\mathrm{h}^{\mathrm{e}-1} \overline{\mathrm{c}} \mathrm{s}_{1} \beta-\frac{\mathrm{a}}{\mathrm{c}} \mathrm{g}\right)
$$

for some $g \in k[h]$. Let $b, r \in k[h]$ be such $g=b c+r$ and $\operatorname{deg} r<M$. Without changing its class in $E^{2}$, we can replace $u$ by $u-\delta\left(\sigma^{-1}\left(p_{1}\right)|H-y \sigma(b)| X\right)$, and then we see that we may assume that

$$
u=\sigma\left(h^{e-1} \bar{c} s_{1} \beta-\frac{a}{c} r\right)\left|Y \wedge X+y \sigma\left(h^{e-1} \bar{c} s_{1} \alpha+\frac{a^{\prime}}{c} r\right)\right| H \wedge X .
$$

If $u$ represents the zero class in $E^{1}$, then there exist $v_{1}, v_{2}, v_{3} \in k[h]$ such that

$$
\begin{aligned}
u & =\delta\left(v_{1} x\left|\mathrm{Y}+v_{2}\right| \mathrm{H}+\mathrm{y} v_{3} \mid \mathrm{X}\right) \\
& =\left(v_{1} \sigma\left(\mathrm{a}^{\prime}\right)+\sigma\left(v_{2}\right)\right) x\left|\mathrm{Y} \wedge \mathrm{H}+\sigma(\mathrm{a})\left(v_{3}-v_{1}\right)\right| \mathrm{Y} \wedge \mathrm{X}-\mathrm{y}\left(v_{3} \sigma\left(\mathrm{a}^{\prime}\right)+\sigma\left(v_{2}\right) \mid \mathrm{H} \wedge \mathrm{X} .\right.
\end{aligned}
$$

Equating coefficients and eliminating $v_{2}$, we see that

$$
\begin{aligned}
& a \sigma^{-1}\left(v_{3}-v_{1}\right)=h^{e-1} \bar{c} s_{1} \beta-\frac{a}{c} r, \\
& -a^{\prime} \sigma^{-1}\left(v_{3}-v_{1}\right)=h^{e-1} \bar{c} s_{1} \alpha+\frac{a^{\prime}}{c} r .
\end{aligned}
$$

Solving now for $s_{1}$ and then for $r$, we see that $u$ must be zero.

Let us show now $u$ represents a non zero element of $E^{2}$. Indeed, if there exists a $p \in k[h]$ such that

$$
u=d(p \mid Y \wedge H \wedge X)=y(1-q \sigma)(p)|H \wedge X-(1-q \sigma)(p) x| Y \wedge H,
$$

then we must have $(1-q \sigma)(p)=0$ and

$$
\begin{aligned}
& \frac{\mathrm{a}}{\mathrm{c}} \mathrm{r}=\mathrm{h}^{\mathrm{e}-1} \overline{\mathrm{c}} s_{1} \beta, \\
& \frac{\mathrm{a}^{\prime}}{\mathrm{c}} \mathrm{r}=-\mathrm{h}^{\mathrm{e}-1} \overline{\mathrm{c}} s_{1} \alpha .
\end{aligned}
$$

Solving these equations for $s_{1}$ and $r$, recalling the way $\alpha$ and $\beta$ were chosen, and using that $\mathrm{h}^{\mathrm{e}-1} \overline{\mathrm{c}} \neq 0$, we see that $\mathrm{s}_{1}=\mathrm{r}=0$.

We conclude in this way that every element of $E_{2,0}^{2}$ is represented uniquely by a cycle of the form (19). In particular, we have a vector space isomorphism $E_{2,0}^{2} \cong \mathcal{S} \oplus k[h] /(c)$.

Homology at $E_{1,0}^{1}$. Let $u=p_{1} x\left|Y+p_{2}\right| H+y p_{3} \mid X \in E_{1,0}^{0}$, with $p_{1}, p_{2}, p_{3} \in k[h]$, an element which survives to $E^{2}$. As $u$ is homologous to $u-\delta\left(\sigma^{-1}\left(p_{1}\right)\right)=\left(p_{2}+a^{\prime} \sigma^{-1}\left(p_{1}\right)\right)\left|H+y\left(p_{3}-p_{1}\right)\right| X$, we can suppose that $p_{1}=0$.

If $u$ is a boundary, so that $u=d\left(f_{1} x\left|Y \wedge H+f_{2}\right| Y \wedge X+y f_{3} \mid H \wedge X\right)+\delta\left(f_{4}\right)$, for some $f_{i} \in k[h]$, looking at the coefficient of $Y$ on both sides of this equality we find that $(1-\sigma)\left(f_{2}\right)+\sigma\left(p_{4}\right)=0$. This implies that $p_{3}=0$ and that $p_{2} \in(1-q \sigma)((c))$. On the other hand, since

$$
d(u)=\sigma(a) p_{3}-a \sigma^{-1}\left(p_{3}\right)=(\sigma-1)\left(a \sigma^{-1}\left(p_{3}\right)\right)=0,
$$

we see that $a \sigma^{-1}\left(p_{3}\right) \in \mathcal{S}$. 
- Suppose first that $\mathrm{q}$ is a root of 1 . Then $\mathrm{p}_{3}=\sigma(\bar{a}) \mathrm{s}$ for some $s \in \mathcal{S}$, according to Proposition 2.1, and thus we have $u=p_{2}|H+y \sigma(\bar{a}) s| X$. In view of the description given above for the boundaries, we conclude that

$$
E_{1,0}^{2} \cong \frac{k[h]}{(1-q \sigma)((c))}|H \oplus y \sigma(\bar{a}) \mathcal{S}| X .
$$

Using Lemma 2.3 we see that the first summand is isomorphic to $k^{\eta(c)} \oplus S$.

- Suppose next that $q$ is not a root of 1 . In this case, since a is not constant, equation (20) implies that $p_{3}=0$. Using again the description of boundaries, we have

$$
E_{1,0}^{2} \cong \frac{k[h]}{(1-q \sigma)((c))} \mid H,
$$

a vector space of dimension $M$.

Homology at $E_{0,0}^{1}$. We have to compute the cokernel of the map $d: A \mid V \rightarrow A$. One sees at once that its image coincides with the image of the map $\psi_{a, 0}: f \in k[h] \mapsto(\sigma-1)(a f) \in k[h]$ from Lemma 2.3. If $q$ is not a root of unity, it is immediate that the classes of $1, \ldots, h^{N-1}$ freely span coker $\psi_{a, 0}$, so that dim $E_{0,0}^{2}=N$. On the other hand, if $q$ is a root of unity, then Lemma 2.3 tells us that the dimension of the cokernel of $\psi_{a, 0}$, equal to that of $E_{0,0}^{2}$, is $\eta(a)=N-\frac{1}{e} \operatorname{deg} \mathcal{N}(a)$.

Proposition 5.6. Let $\mathrm{r} \neq 0$. According to whether $\mathrm{r}$ is regular or not, there are isomorphisms of $\mathcal{S}$-modules

or

$$
\mathrm{HH}_{\mathrm{p}}(A)^{(\mathrm{r})} \cong \mathrm{E}_{\mathrm{p}, 0}^{2(\mathrm{r})} \cong \begin{cases}\mathcal{S}, & \text { if } \mathrm{p}=0 \\ k, & \text { if } \mathrm{p}=1 \\ 0, & \text { if } \mathrm{p}=2\end{cases}
$$

$$
\mathrm{HH}_{\mathfrak{p}}(A)^{(r)} \cong \mathrm{E}_{\mathrm{p}, 0}^{2(\mathrm{r})} \cong \begin{cases}\mathcal{S}, & \text { if } \mathrm{p}=0 \\ \mathcal{S} \oplus \mathcal{S}, & \text { if } \mathrm{p}=1 \\ \mathcal{S}, & \text { if } \mathrm{p}=2\end{cases}
$$

Proof. By symmetry, we can consider just the case where $r>0$.

Homology at $E_{2,0}^{1(r)}$. Let $u \in E_{2,0}^{O(r)}$ be an element representing a cycle in $E^{1}$. It follows that $u=$ $y^{r-1} p_{1}\left|Y \wedge H+y^{r} p_{2}\right| Y \wedge X+y^{r+1} p_{3} \mid H \wedge X$ with $p_{1}, p_{2}, p_{3} \in k[h]$. Without loss of generality, we can assume that $p_{2}=p_{3}=0$; if that is not the case, we can replace $u$ by

$$
u+\delta\left(y^{r-1} \sigma^{-1}\left(p_{2}\right)\left|Y+y^{r} \sigma^{-1}\left(p_{3}\right)\right| H\right)
$$

without changing the class of $u$ in $E^{1}$. Computing, we find then that

$$
d(u)=y^{r-1}\left(1-q^{r}\right) p_{1} h\left|Y+y^{r}\left(p_{1}-q \sigma\left(p_{1}\right)\right)\right| H .
$$

Comparing with equation (9a) we see that, since $d(u)$ is in the image of $\delta,\left(1-q^{r}\right) p_{1}=0$ and $p_{1}-q \sigma\left(p_{1}\right)=0$. If $r$ is a regular weight, it follows that $p_{1}=0$, so $E_{2,0}^{2(r)}=0$. On the other hand, if $r$ is singular, these equations are satisfied if and only if $p_{1} \in \mathrm{h}^{e-1} \mathcal{S}$ : in this case we have $\mathrm{E}_{2,0}^{2(\mathrm{r})} \cong \mathrm{h}^{\mathrm{e}-1} \mathcal{S}$. 
Homology at $E_{1,0}^{1(r)}$. Let $u=y^{r-1} p_{1}\left|Y+y^{r} p_{2}\right| H+y^{r+1} p_{3} \mid X \in E_{1,0}^{O(r)}$, with $p_{1}, p_{2}, p_{3} \in k[h]$, an element which lives to $E^{2}$. Up to replacing $u$ by $u-\delta\left(y^{r} \sigma^{-1}\left(p_{3}\right)\right)$, we can assume that $p_{3}=0$, so that

$$
d(u)=y^{r}\left(p_{1}-\sigma\left(p_{1}\right)+\left(q^{r}-1\right) h p_{2}\right)=0 .
$$

Assume that $r$ is singular. It follows that $p_{1} \in \mathcal{S}$; moreover, in view of formula (21), we can reduce $p_{2}$ modulo the image of $1-q \sigma$, so that we can suppose that $p_{2} \in h^{e-1} \mathcal{S}$. From equations (8d), (8e), (8f) and (9a) we see then that $u$ is not a boundary and we conclude that $\mathrm{E}_{1,0}^{2(\mathrm{r})} \cong \mathcal{S} \oplus \mathcal{S}$ in this case, freely generated as a $\mathcal{S}$-module by the classes of $y^{r-1} \mid \mathrm{Y}$ and $\mathrm{y}^{\mathrm{r}} \mathrm{h}^{\mathrm{e}-1} \mid \mathrm{H}$.

Finally, let us assume that $r$ is regular. Using again (21), we see that we can now replace $u$ by an homologous element of the same form but now with $p_{1} \in k$ and then, because of (22), we must have $p_{2}=0$. In this way, we see that $u$ must be a scalar multiple of $y^{r-1} \mid Y$. If such an element is a boundary, looking at the constant term in the formulas (8d), (8e), (8f) and (9a), we infer that $u$ is zero, therefore $E_{1,0}^{2(r)}$ is one-dimensional.

Homology at $E_{0,0}^{1(r)}$. Let $u=y^{r} p \in E_{0,0}^{O(r)}$. We can add to $u$ elements in the image of $d$ without changing its homology class; doing so, we can assume that $p \in S$. Moreover, $u$ itself is then not in the image of $d$ : this means that $E_{0,0}^{1(r)} \cong S$, freely generated by the class of 1 .

\section{Hochschild cohomology}

In this section we compute the Hochschild cohomology of $A$ using, as before, a spectral sequence. Write $\widehat{V}=\operatorname{hom}_{k}(V, k)$, and let $\{\hat{\mathrm{Y}}, \hat{\mathrm{A}}, \widehat{X}\}$ be the basis of $\widehat{\nabla}$ dual to $\{Y, H, X\}$. We identify in the usual way hom ${ }_{k}\left(\wedge^{p} \vee, k\right)$ with $\Lambda^{p} \widehat{V}$. Applying the functor hom $\operatorname{A}^{e}(-, A)$ to the resolution constructed in 4.3 we obtain a double complex whose cohomology is the Hochschild cohomology $\mathrm{HH}^{\bullet}(A)$ of $A$. After we identify hom $A^{e}\left(A\left|\Lambda^{p} V\right| A, A\right)$ with $A \mid \Lambda^{p} \hat{V}$ in the natural way, this double complex is

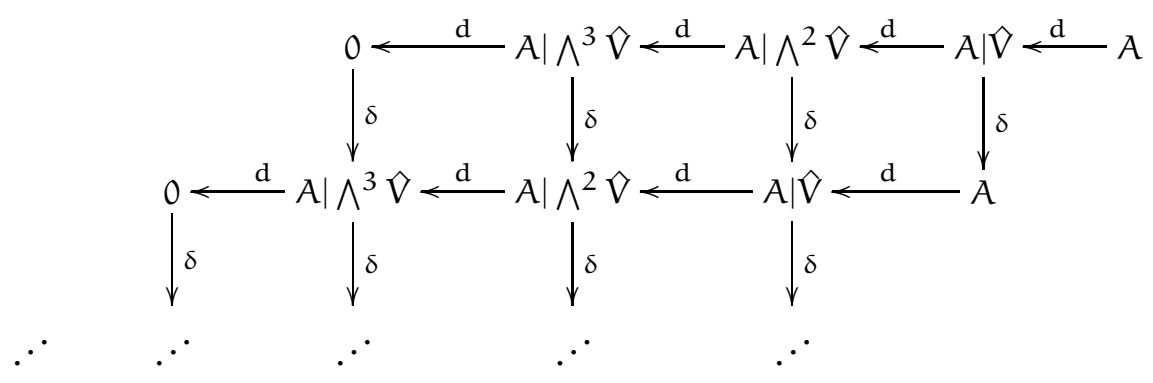

with differentials given by

$$
\begin{aligned}
& d(u)=[u, y]|\hat{Y}+[u, h]| \hat{H}+[u, x] \mid \hat{X} ; \\
& \mathrm{d}(\mathrm{u} \mid \hat{\mathrm{Y}})=[\mathrm{h}, \mathrm{u}]_{\mathrm{q}}|\hat{\mathrm{P}} \wedge \hat{\mathrm{H}}-[\mathrm{u}, \mathrm{x}]| \hat{\mathrm{Y}} \wedge \hat{\mathrm{X}} ; \\
& \mathrm{d}(\mathrm{u} \mid \hat{\mathrm{H}})=[\mathrm{x}, \mathrm{u}]_{\mathbf{q}}\left|\hat{\mathrm{H}} \wedge \hat{\mathrm{X}}-\sum_{i} \lambda_{i} \int_{i} h^{\mathrm{s}} u h^{\mathrm{t}}\right| \hat{\gamma} \wedge \hat{X}+[u, y]_{\mathbf{q}} \mid \hat{\gamma} \wedge \hat{\mathrm{A}} ; \\
& \mathrm{d}(\mathrm{u} \mid \hat{\mathrm{X}})=[\mathrm{u}, \mathrm{h}]_{\mathbf{q}}|\hat{\mathrm{H}} \wedge \hat{\mathrm{X}}+[\mathrm{u}, \mathrm{y}]| \hat{\mathrm{Y}} \wedge \hat{\mathrm{X}}
\end{aligned}
$$




$$
\begin{aligned}
& d(u \mid \hat{P} \wedge \hat{A})=-[x, u]_{q} \mid \hat{P} \wedge \hat{A} \wedge \hat{X} \\
& d(u \mid \hat{\gamma} \wedge \hat{X})=q[h, u] \mid \hat{Y} \wedge \hat{H} \wedge \hat{X} ; \\
& d(u \mid \hat{H} \wedge \hat{X})=[u, y]_{q} \mid \hat{P} \wedge \hat{H} \wedge \hat{X} ;
\end{aligned}
$$

and

$$
\begin{aligned}
& \delta(u \mid \hat{\gamma})=u x ; \\
& \delta(u \mid \hat{A})=-\sum_{i} \alpha_{i} \int_{i} h^{s} u h^{t} ; \\
& \delta(u \mid \hat{X})=y u ; \\
& \delta(u \mid \hat{\gamma} \wedge \hat{H})=\sum_{i} \alpha_{i} \int_{i} q^{t} h^{s} u h^{t}|\hat{\gamma}+u x| \hat{H} ; \\
& \delta(u \mid \hat{\gamma} \wedge \hat{X})=u x|\hat{X}-y u| \hat{\gamma} ; \\
& \delta(u \mid \hat{H} \wedge \hat{X})=-y u\left|\hat{H}-\sum_{i} \alpha_{i} \int_{i} q^{s} h^{s} u h^{t}\right| \hat{X} ; \\
& \delta(u \mid \hat{\gamma} \wedge \hat{H} \wedge \hat{X})=u x\left|\hat{H} \wedge \hat{X}+\sum_{i} \alpha_{i} \int_{i} q^{i-1} h^{s} u h^{t}\right| \hat{\gamma} \wedge \hat{X}+y u \mid \hat{\gamma} \wedge \hat{H} ;
\end{aligned}
$$

We consider the spectral sequence $E$ which arises from the filtration of this double complex by columns.

\subsection{First Page}

In this section we deal with the first page of the spectral sequence. Let $y$ be the complex

$$
0 \longrightarrow A\left|\Lambda^{3} \widehat{\nabla} \stackrel{\delta}{\longrightarrow} A\right| \Lambda^{2} \widehat{\nabla} \stackrel{\delta}{\longrightarrow} A \mid \widehat{\nabla} \stackrel{\delta}{\longrightarrow} A
$$

with differentials as in (24a)-(24g). As before, we have $E_{1}^{p, q} \cong H^{p-q}(y)$ for all $q>0$ and the vector spaces $E_{1}^{p, 0}$ are isomorphic to the kernels of the differentials of $y$. For each $r \in \mathbb{Z}$ we denote $y_{(r)}$ the component of weight $r$ in $y$, and extend this notation to related objects.

Proposition 6.1. If $r \in \mathbb{Z}$ is non zero, then the complex $y_{(r)}$ is exact. On the other hand, there are $\mathcal{S}$-module isomorphisms

$$
H^{p}\left(y_{(0)}\right) \cong \begin{cases}k[h] /(c), & \text { if } 0 \leq p \leq 1 ; \\ 0, & \text { otherwise. }\end{cases}
$$

Proof. We prove this by computing the relevant homology groups:

- If $u=p \mid \hat{Y} \wedge \hat{H} \wedge \hat{X} \in y_{(0)}^{3}$ with $p \in k[h]$, then

$$
\delta(u)=p x\left|\hat{\mathrm{A}} \wedge \hat{\mathrm{X}}+p \sigma\left(\mathrm{a}^{\prime}\right)\right| \hat{\mathrm{Y}} \wedge \hat{\mathrm{X}}+\mathrm{yp} \mid \hat{\mathrm{P}} \wedge \hat{\mathrm{H}} .
$$

It is clear then that $\mathrm{H}^{3}\left(y_{(0)}\right)=0$.

- Let $u=y p_{1}\left|\hat{\gamma} \wedge \hat{H}+p_{2}\right| \hat{\gamma} \wedge \hat{X}+p_{3} x \mid \hat{A} \wedge \hat{X} \in y_{(0)}^{2}$ with $p_{1}, p_{2}, p_{3} \in k[h]$. One can see that

$$
\delta(u)=y\left(p_{1} \sigma\left(a^{\prime}\right)-p_{2}\right)\left|\hat{\gamma}+\left(a \sigma^{-1}\left(p_{1}-p_{3}\right)\right)\right| \hat{A}+\left(-p_{3} \sigma\left(a^{\prime}\right)+p_{2}\right) x \mid \hat{X} .
$$

In particular, if $u$ is a cycle, $p_{2}=\sigma\left(a^{\prime}\right) p_{1}$ and $p_{3}=p_{1}$. Comparing with the expression (26) for 2-boundaries in $y$, we see at once that $\mathrm{H}^{2}\left(y_{(0)}\right)=0$. 
- Finally, let $u=y p_{1}\left|\hat{\gamma}+p_{2}\right| \hat{A}+p_{3} x \mid \hat{X} \in y_{(0)}^{1}$, with $p_{1}, p_{2}, p_{3} \in k[h]$, a 1-cycle. Since we can replace $u$ for $u+\delta\left(p_{1} \mid \hat{\mathrm{H}}\right)$, without changing the homology class it represents, we can assume that $p_{1}=0$, and then $\delta(u)=a \sigma^{-1}\left(p_{3}\right)-p_{2} a^{\prime}=0$. It follows that there exists $g \in k[h]$ such that $p_{3}=\sigma\left(\frac{a^{\prime}}{c} g\right)$ and $p_{2}=\frac{a}{c} g$. Let $b, r \in k[h]$ such that $g=b c+r$ and $\operatorname{deg} r<M$. Then

$$
u+\delta(\sigma(b) x \mid \hat{\mathrm{A}} \wedge \hat{\mathrm{X}})=\frac{\mathrm{a}}{\mathrm{c}} \mathrm{r}\left|\hat{\mathrm{A}}+\sigma\left(\frac{\mathrm{a}^{\prime}}{\mathrm{c}} \mathrm{r}\right) x\right| \hat{\mathrm{X}}
$$

This means that all classes in $H^{1}\left(y_{(0)}\right)$ can be represented by a element of the form $\frac{a}{c} r \mid \hat{H}+$ $\sigma\left(\frac{a^{\prime}}{c} r\right) x \mid \hat{X}$ with $r \in k[h]$ and $\operatorname{deg} r<M$ and, moreover, such an element represents the zero class only when it is itself zero: this can be seen by looking at the degree of the coefficient of $\hat{\mathrm{H}}$ appearing the formula (27) for 1-boundaries. Conversely, every such element is a cycle. We conclude that $H^{1}\left(y_{(0)}\right) \cong k[h] /(c)$.

- If $u=y p_{1}\left|\hat{\gamma}+p_{2}\right| \hat{H}+p_{3} x \mid \hat{\chi} \in y_{(0)}^{1}$, with $p_{1}, p_{2}, p_{3} \in k[h]$, then $\delta(u)=a \sigma^{-1}\left(p_{1}+p_{3}\right)-$ $p_{2} a^{\prime}$, so $H^{0}\left(y_{(0)}\right) \cong k[h] /(c)$.

It remains to check, in these last two items, that the obtained isomorphisms are $\mathcal{S}$-linear: this is just a matter of following the computation, and we omit the details.

Let us now fix $r>0$.

- If $u=y^{r} p \mid \hat{\gamma} \wedge \hat{A} \wedge \hat{X} \in y_{(r)}^{3}$, with $p \in k[h]$, then

$$
\delta(u)=y^{r+1} p\left|\hat{\gamma} \wedge \hat{A}+y^{r} p \sum_{i} \alpha_{i} q^{i-1}[i]_{q^{r}} h^{i-1}\right| \widehat{\gamma} \wedge \hat{X}+y^{r-1} a \sigma^{-1}(p) \mid \hat{H} \wedge \hat{X} .
$$

Looking at the coefficient of $\widehat{\gamma} \wedge \hat{\mathrm{H}}$ we see that $u$ is a cycle if and only if $u$ is zero, so $\mathrm{H}^{3}\left(y_{(r)}\right)=0$.

- Let $u=y^{r+1} p_{1}\left|\hat{\gamma} \wedge \hat{A}+y^{r} p_{2}\right| \hat{\gamma} \wedge \hat{X}+y^{r-1} p_{3} \mid \hat{H} \wedge \hat{X} \in y_{(r)}^{2}$, with $p_{1}, p_{2}, p_{3} \in k[h]$. Since

$$
\begin{aligned}
\delta(u)=y^{r+1}\left(p_{1} \sum_{i} \alpha_{i} q^{i-1}[i]_{q^{r}} h^{i-1}-p_{2}\right) \mid \hat{\gamma} & +y^{r}\left(a \sigma^{-1}\left(p_{1}\right)-p_{3}\right) \mid \hat{H} \\
& +y^{r-1}\left(a \sigma^{-1}\left(p_{2}\right)-p_{3} \sum_{i} \alpha_{i}[i]_{q^{r}} h^{i-1}\right) \mid \hat{X},
\end{aligned}
$$

it is easy to see that $u$ is a cycle if and only if

$$
\begin{aligned}
& p_{2}=p_{1} \sum_{i} \alpha_{i} q^{i-1}[i]_{q^{r}} h^{i-1}, \\
& p_{3}=a \sigma^{-1}\left(p_{1}\right),
\end{aligned}
$$

and in that case, according to (28), we have $u=\delta\left(y^{r} p_{1} \mid \hat{\gamma} \wedge \hat{H} \wedge \hat{X}\right)$. We conclude that $\mathrm{H}^{2}\left(y_{(r)}\right)=0$.

- Let $u=y^{r+1} p_{1}\left|\hat{\gamma}+y^{r} p_{2}\right| \hat{A}+y^{r-1} p_{3} \mid \hat{X} \in y_{(r)}^{1}$, with $p_{1}, p_{2}, p_{3} \in k[h]$ a cycle. Without changing its homology class, we can replace $u$ by $u+\delta\left(y^{r} p_{1}\left|\hat{\gamma} \wedge \hat{X}+y^{r-1} p_{2}\right| \hat{H} \wedge \hat{X}\right)$, and hence we can suppose that $p_{1}=p_{2}=0$. In that case $\delta(u)=y^{r} p_{3}$, and we see that $u=0$. It follows that $\mathrm{H}^{1}\left(\mathrm{y}_{(\mathrm{r})}\right)=0$.

- Finally, for each $p \in k[h], \delta\left(y^{r-1} p \mid \hat{X}\right)=y^{r} p$, so that $\delta\left(y_{(r)}^{1}\right)=y_{(r)}^{0}$ and $H^{0}\left(y_{(r)}\right)=0$. 
Corollary 6.2. If $r \in \mathbb{Z}$, the dimensions of the vector spaces appearing in the component $\mathrm{E}_{1(\mathrm{r})}$ of $\mathrm{E}_{1}$ are

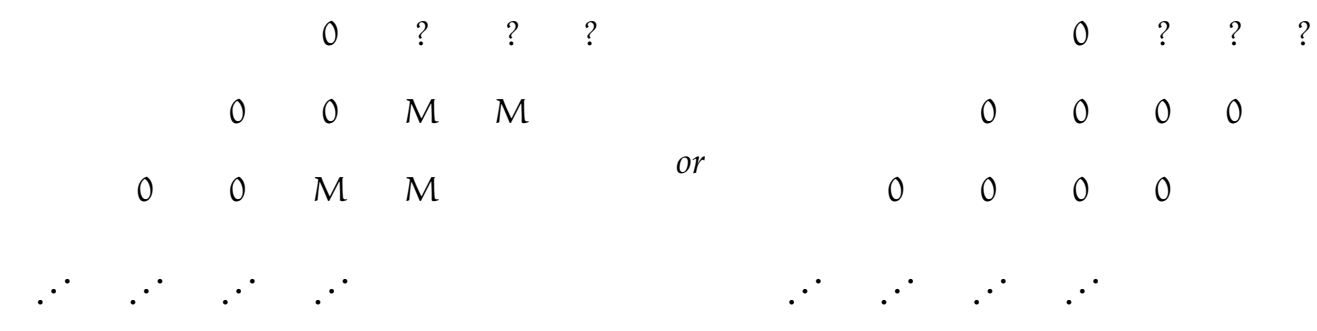

if $\mathrm{r}=0$ or $\mathrm{r} \neq 0$, respectively. The question marks denote vector spaces for which we still do not know the dimension.

Proof. This follows from the proposition and the isomorphisms $E_{1(r)}^{p, q} \cong H^{p-q}\left(y_{(r)}\right)$.

\subsection{The second page}

Proposition 6.3. For each $\mathrm{p} \geq 0$, the differential $\mathrm{d}_{1}^{\mathrm{p}, \mathrm{p}}: \mathrm{E}_{1}^{\mathrm{p}, \mathrm{p}} \rightarrow \mathrm{E}_{1}^{\mathrm{p}+1, \mathrm{p}}$ vanishes. The page $\mathrm{E}_{\infty}$ then coincides with $\mathrm{E}_{1}$, except at the places marked with question marks in the diagrams of Corollary 6.2, and we have

$$
H H^{p}(A)_{(r)} \cong \begin{cases}k[h] /(c), & \text { if } r=0 \\ 0, & \text { if } r \neq 0 .\end{cases}
$$

Proof. The set of homology classes of the elements of $\left\{h^{l}: 0 \leq l<M\right\}$ is a basis of the space $E_{1}^{p, p}$, and

$$
d\left(h^{l}\right)=\left(q^{l}-1\right) y h^{l}\left|\hat{\gamma}-\left(q^{l}-1\right) h^{l} x\right| \hat{X}=\delta\left(-\left(q^{l}-1\right) h^{l} \mid \hat{\gamma} \wedge \hat{\chi}\right) .
$$

It follows that $d_{1}^{p, p}$ is indeed zero, as claimed. The rest of the proposition is then a consequence of the fact that the spectral sequence $E$ converges to $\mathrm{HH}^{\bullet}(A)$.

Proposition 6.4. If $\mathrm{q}$ is a root of unity, then

$$
E_{2(0)}^{p, 0} \cong \begin{cases}\mathcal{S}, & \text { if } \mathrm{p}=0 ; \\ \mathcal{S} \oplus \mathcal{S}, & \text { if } \mathrm{p}=1 ; \\ \mathcal{S} \oplus \mathrm{k}^{\eta(\mathrm{a} / \mathrm{c})}, & \text { if } \mathrm{p}=2,\end{cases}
$$

where, as in Lemma 2.3, $\eta(\mathrm{a} / \mathrm{c})=\mathrm{N}-\mathrm{M}-\operatorname{deg} \mathcal{N}(\mathrm{a} / \mathrm{c}) / \mathrm{e}$, and if $\mathrm{q}$ has infinite order,

$$
E_{2(0)}^{p, 0} \cong \begin{cases}k, & \text { if } p=0 \\ k, & \text { if } p=1 \\ k^{N-M,}, & \text { if } p=2\end{cases}
$$

Proof. We write, during this proof, $\mathrm{E}_{\mathrm{r}}^{\mathrm{p}, \mathrm{q}}$ instead of $\mathrm{E}_{\mathrm{r}(0)}^{\mathrm{p}, \mathrm{q}}$ for simplicity. 
Homology at $E_{1}^{0,0}$. If $u=p \in E_{1}^{0,0}$, so that in fact $p \in k[h]$, we have

$$
d(p)=y(\sigma(p)-p)|\widehat{\gamma}-(\sigma(p)-p) x| \widehat{X}
$$

It follows that $\mathrm{E}_{1}^{0,0}=\operatorname{ker}(\sigma-1)=\mathcal{S}$.

Homology at $E_{1}^{1,0}$. If $u \in E_{1}^{1,0}$, there exist $p_{1}, p_{2} \in k[h]$ such that $u=y p_{1}\left|\widehat{Y}+\frac{a}{d} p_{2}\right| \hat{H}+\left(\sigma\left(\frac{a^{\prime}}{d} p_{2}\right)-\right.$ $\left.p_{1}\right) x \mid \hat{X}$; this is a consequence of the formulas (24a), (24b) and (24c) using the same reasoning as in the third step of the proof of Proposition 6.1. Moreover, there exist $s_{1} \in \mathcal{S}$ and $b \in k[h]$ such that $p_{1}=s_{1}+(\sigma-1)(b)$ and we can replace $u$ by $u-d(b)$ so, in the end, we can assume that $p_{1}=s_{1} \in \mathcal{S}$. In that case, $u$ is boundary only if it zero: this follows by comparing with the coefficient of $\widehat{\gamma}$ in (29). Computing, we find that

$$
\begin{aligned}
\mathrm{d}(\mathrm{u})=(\sigma-\mathrm{q})\left(\frac{\mathrm{a}}{\mathrm{c}} \mathrm{p}_{2}\right) x\left|\hat{\mathrm{A}} \wedge \hat{\mathrm{X}}+\mathrm{y}(\sigma-\mathrm{q})\left(\frac{\mathrm{a}}{\mathrm{c}} \mathrm{p}_{2}\right)\right| \widehat{P} \wedge \hat{\mathrm{A}} & \\
& +\left((\sigma-1)\left(\frac{\mathrm{aa^{ \prime }}}{\mathrm{c}} \mathrm{p}_{2}\right)-\frac{\mathrm{a}}{\mathrm{c}} \mathrm{p}_{2}\left(\mathrm{q} \sigma\left(\mathrm{a}^{\prime}\right)-\mathrm{a}^{\prime}\right)\right) \mid \hat{\mathrm{P}} \wedge \hat{\mathrm{X} .}
\end{aligned}
$$

If $d(u)=0$, then $(\sigma-q)\left(\frac{a}{c} p_{2}\right)=0$ and $\frac{a}{c} p_{2} \in h \mathcal{S}$; conversely, if $\frac{a}{c} p_{2} \in h \mathcal{S}$, then $u$ is a cycle. we treat separately two cases, according to whether $\mathrm{q}$ is a root of unity or not.

- Suppose first that $q$ is not a root of 1 . As $\frac{a}{c} p_{2} \in h \mathcal{S}$ and $\mathcal{S}=k$, then $p_{2} \in k$. Evaluating $\frac{a}{c} p_{2}$ at zero, and using the hypothesis that $a(0) \neq 0$, we see that $p_{2}=0$. In this case, then, $u$ is a scalar multiple of $y|\hat{Y}-x| \hat{X}$. Since all such non zero multiples are cycles and not boundaries, we conclude that $E_{2}^{1,0}$ is one dimensional, generated by the class of $y|\hat{\gamma}-x| \hat{X}$.

- Suppose now that $q$ is a root of 1 . As $h \nmid a$, we must have $h \mid p_{2}$ and $\frac{a}{c} \frac{p_{2}}{h} \in \mathcal{S}$. There exists then, by Proposition 2.1(i), $s_{2} \in \mathcal{S}$ such that $\mathrm{p}_{2}=\mathrm{hs} \mathrm{s}_{2} \overline{\left(\frac{\mathrm{a}}{\mathrm{c}}\right)}$. This gives us a description of homology: it is the free $\mathcal{S}$-module of rank 2 generated by the classes of $y|\hat{\gamma}-x| \hat{X}$ and $\mathcal{N}\left(\frac{\mathrm{a}}{\mathrm{c}}\right) h\left|\hat{\mathrm{A}}+\sigma\left(\frac{\mathrm{a}^{\prime}}{\mathrm{c}} \overline{\mathrm{a}} \mathrm{c} h\right) x\right| \hat{\chi}$.

Homology at $E_{1}^{2,0}$. Let $u \in E_{1}^{2,0}$, so in fact $u \in E_{0}^{2,0}$ and $\delta(u)=0$. In view of (27), there exists $p \in k[h]$ such that $u=y p\left|\hat{\gamma} \wedge \hat{\mathrm{A}}+p \sigma\left(a^{\prime}\right)\right| \hat{\gamma} \wedge \hat{X}+p x \mid \hat{\mathrm{A}} \wedge \hat{\mathrm{X}}$.

The element $u$ is a boundary if there exist $f_{1}, f_{2} \in k[h]$ such that $u=d\left(y f_{1}\left|\hat{\gamma}+\frac{a}{c} f_{2}\right| \hat{A}+\right.$ $\left.\left(\sigma\left(\frac{a^{\prime}}{c} f_{2}\right)-f_{1}\right) x \mid \hat{X}\right)$ or, making this explicit,

$$
\begin{aligned}
& p=(\sigma-q)\left(\frac{a}{c} f_{2}\right), \\
& \sigma\left(a^{\prime}\right) p=D_{q}\left(\frac{a a^{\prime}}{c} f_{2}\right)-\frac{a}{c} f_{2}\left(q \sigma\left(a^{\prime}\right)-a^{\prime}\right) .
\end{aligned}
$$

The second equation follows from the first, and we conclude that $u$ is a boundary if and only if $p \in \operatorname{im} \psi_{a / c, 1}$ with $\psi_{a / c, 1}$ defined as in Lemma 2.3. In other words, there is an isomorphism $\mathrm{E}_{2}^{2,0} \cong$ coker $\psi_{\mathrm{a} / \mathrm{c}, 1}$. We have two cases:

- First, suppose that $q$ is not a root of 1 . If $\operatorname{deg}\left(\frac{a}{c}\right)>1$, then $\operatorname{deg} \psi_{a / c, 1}(f)=\operatorname{deg}\left(\frac{a}{c}\right)+\operatorname{deg}(f)$ for $f \in k[h] \backslash 0$. It follows then that coker $\psi_{a / c, 1}$ is freely spanned by the classes of $1, h, \ldots$, $h^{\mathrm{N}-\mathrm{M}-1}$, because $\operatorname{im} \psi_{\mathrm{a} / \mathrm{c}, 1}$ is spanned by a set of polynomials of each degree greater or equal to $N-M$. We conclude that $\operatorname{dim}\left(\operatorname{coker}\left(\psi_{\mathrm{a} / \mathrm{c}, 1}\right)\right)=\mathrm{N}-\mathrm{M}$.

On the other hand, if $\operatorname{deg}\left(\frac{a}{c}\right)=1$, we have $\operatorname{deg} \psi_{a / c, 1}(f)=1+\operatorname{deg}(f)$ for all non-constant $f \in k[h]$ and $\operatorname{deg} \psi_{a / c, 1}(f)=0$ for $f \in k \backslash 0$, so that the cokernel is freely spanned by the class of $h$. In particular, $\operatorname{dim} \operatorname{coker}\left(\psi_{\mathrm{a} / \mathrm{c}, 1}\right)=1=\mathrm{N}-\mathrm{M}$. 
- Suppose now that $\mathrm{q}$ is a root of 1 . We computed the dimension of coker $\psi_{\mathrm{a} / \mathrm{c}, 1}$ in Lemma 2.3, so that the the dimension of $E_{2(0)}^{2,0}$ is $\eta(a / c)$, as claimed in the statement of the proposition.

Corollary 6.5. If $\mathrm{q}$ is a root of unity, then there are isomorphisms of $\mathcal{S}$-modules

$$
\mathrm{HH}^{\mathrm{p}}(\mathrm{A})_{(\mathrm{O})} \cong \begin{cases}\mathcal{S}, & \text { if } \mathrm{p}=0 ; \\ \mathcal{S} \oplus \mathcal{S}, & \text { if } \mathrm{p}=1 ; \\ \mathcal{S} \oplus \mathrm{k}^{\mathfrak{\eta}(\mathrm{a} / \mathrm{c})} \oplus \mathrm{k}[\mathrm{h}] /(\mathrm{c}), & \text { if } \mathrm{p}=2 .\end{cases}
$$

If, on the other hand, q has infinite order,

$$
H H^{p}(A)_{(0)} \cong \begin{cases}k, & \text { if } p=0 ; \\ k, & \text { if } p=1 ; \\ k^{N-M} \oplus k^{M}, & \text { if } p=2 .\end{cases}
$$

Proof. This follows from the proposition and the convergence of the spectral sequence.

Remark 6.6. In the computation of the Hochschild cohomology the fact that $a(0) \neq 0$ is only used in the proof of the Proposition 6.4. In the case when $\mathrm{q}$ is not a root of 1 , using an analogous reasoning one can prove that if $a(0)=0$ and $a \neq h^{N}$ then the same result holds. If instead $a=h^{N}$ then

$$
E_{2(0)}^{p, 0} \cong \begin{cases}k, & \text { if } p=0 \\ k^{2}, & \text { if } p=1 \\ k^{N-M+1,} & \text { if } p=2\end{cases}
$$

On the other hand, if $\mathrm{q}$ is a root of 1 then

$$
\mathrm{E}_{2(0)}^{\mathrm{p}, 0} \cong \begin{cases}\mathcal{S}, & \text { if } \mathrm{p}=0 ; \\ \mathcal{S} \oplus \mathcal{S}, & \text { if } \mathrm{p}=1 ; \\ \mathcal{S} \oplus \mathrm{k}^{\eta(\mathrm{a} /(\mathrm{ch}))+1} & \text { if } \mathrm{p}=2\end{cases}
$$

This difference is to be expected because, for example, when $a=h^{N}$ we have gradings on $A$ such that $\operatorname{deg} h=1$ and $\operatorname{deg} x+\operatorname{deg} y=N$. The eulerian derivation induced by one of these gradings is a non zero class in $\mathrm{HH}^{1}(\mathrm{~A})$, which is not cohomologous to the induced by the weight.

Proposition 6.7. Let $r \neq 0$. According to whether $r$ is regular or not, there are isomorphisms of $\mathcal{S}$-modules

or

$$
E_{2(r)}^{p, 0} \cong \begin{cases}0, & \text { if } p=0 \\ 0, & \text { if } p=1 \\ 0, & \text { if } p=2\end{cases}
$$

$$
\mathrm{E}_{2(\mathrm{r})}^{\mathrm{p}, 0} \cong \begin{cases}\mathcal{S}, & \text { if } \mathrm{p}=0 \\ \mathcal{S} \oplus \mathcal{S}, & \text { if } \mathrm{p}=1 \\ \mathcal{S}, & \text { if } \mathrm{p}=2\end{cases}
$$


Proof. Homology at $E_{1(r)}^{0,0}$. Let $u \in E_{0(r)}^{0,0}$, so that $u=y^{r} p$ for some $p \in k[h]$. Since

$$
d(u)=y^{r+1}(\sigma(p)-p)\left|\hat{\gamma}+\left(1-q^{r}\right) p h\right| \hat{A}+y^{r-1}\left(a \sigma^{-1}(p)-\sigma^{r}(a) p\right) \mid \hat{X},
$$

$u$ is a non zero cycle if and only if $r$ is a singular weight and $p \in \mathcal{S}$.

Homology at $E_{1(r)}^{1,0}$. If $u \in E_{1(r)}^{1,0}$, then there exist $p_{1}, p_{2}, p_{3} \in k[h]$ such that $u=y^{r+1} p_{1} \mid \hat{\gamma}+$ $y^{r} p_{2}\left|\hat{A}+y^{r-1} p_{3}\right| \hat{X}$ and $\delta(u)=0$. This condition implies immediately, using (24a), (24b) and (24c), that $p_{3}=p_{2} \sum_{i} \alpha_{i}[i]_{q} h^{i-1}-a \sigma^{-1}\left(p_{1}\right)$. Let us suppose now that $d(u)=0$.

- If $r$ is regular, we can replace $u$ by $u-d\left(\left(1-q^{r}\right)^{-1} y^{r}\left(p_{2}-p_{2}(0)\right) / h\right)$ without changing its homology class, and this amounts to assuming initially that $p_{2} \in \mathrm{k}$. In that case, it is easy to see that the coefficient of $\hat{\gamma} \wedge \hat{H}$ in $d(u)$ is $y^{r+1}\left(q\left(q^{r}-1\right) h p_{1}+(1-q) p_{2}\right)=0$ and, then, $p_{1}=p_{2}=0$. Similarly, looking at the coefficient of $\hat{\mathrm{A}} \wedge \hat{\mathrm{X}}$, we can conclude that $\mathrm{p}_{3}=0$.

- If $r$ is singular, there exist $b \in k[h]$ and $s_{1} \in S$ such that $p_{1}=\sigma(b)-b+s_{1}$; by replacing $u$ by $u-d\left(y^{r} b\right)$, which we may do as it does not change the homology class, we may assume that $p_{1}=s_{1} \in S$. Computing, we find that

$$
d(u)=y^{r+1}(\sigma-q)\left(p_{2}\right)\left|\hat{\gamma} \wedge \hat{A}+y^{r} \sigma\left(a^{\prime}\right)(\sigma-q)\left(p_{2}\right)\right| \hat{\gamma} \wedge \hat{X}+y^{r-1} a(\sigma-q)\left(\sigma^{-1}\left(p_{2}\right)\right) \mid \hat{H} \wedge \hat{X},
$$

and it is clear that this vanishes exactly when $p_{2} \in h \mathcal{S}$. We see that every element of $E_{2(r)}^{1,0}$ is represented by an element in the $\mathcal{S}$-submodule generated by the elements

$$
y^{r+1}\left|\hat{\gamma}-y^{r-1} a\right| \hat{X} \quad y^{r} h\left|\hat{A}+y^{r-1} a^{\prime} h\right| \hat{X} .
$$

Comparing with (30), it is easy to see that this submodule does not contain non zero boundaries, so $E_{2(r)}^{1,0}$ is $\mathcal{S}$-free of rank 2 .

Homology at $E_{1(r)}^{2,0}$. Let $u=y^{r+1} p_{1}\left|\hat{\gamma} \wedge \hat{A}+y^{r} p_{2}\right| \hat{\gamma} \wedge \hat{X}+y^{r-1} p_{3} \mid \hat{A} \wedge \hat{X} \in E_{1(r)}^{2,0}$.

- If $r$ is regular, let $b_{i}=\left(p_{i}-p_{i}(0)\right)\left(q\left(q^{r}-1\right) h\right)^{-1}$ for $i \in\{1,3\}$. We may replace $u$ by $u-$ $d\left(b_{1}\left|\hat{Y}+b_{3}\right| \hat{X}\right)$, and a computation using (23b) and (23d) shows that this means that we can assume that $p_{1}, p_{3} \in k$. Using now (24d), (24e) and (24f), we easily see that $\delta(u)=0$ if and only if $u=0$. It follows that in this case $E_{2(r)}^{2,0}=0$.

- To finish, suppose next that $r$ is singular. Since

$$
\delta(u)=y^{r+1}\left(\sigma\left(a^{\prime}\right) p_{1}-p_{2}\right)\left|\hat{\gamma}+y^{r}\left(a \sigma^{-1}\left(p_{1}\right)-p_{3}\right)\right| \hat{A}+y^{r-1}\left(a \sigma^{-1}\left(p_{2}\right)-a^{\prime} p_{3}\right) \mid \hat{X}=0,
$$

we see that $p_{3}=a \sigma^{-1}\left(p_{1}\right)$ and $p_{2}=\sigma\left(a^{\prime}\right) p_{1}$. If $b \in k[h]$ and $s \in S$ are such that $p_{1}=$ $\sigma(b)-q b+h s$, we can replace $u$ by $u-d\left(y^{r} b\left|\hat{H}+y^{r-1} b a^{\prime}\right| \hat{X}\right)$, which is

$$
\mathrm{y}^{\mathrm{r}+1} h \mathrm{~s}_{1}\left|\hat{\mathrm{Y}} \wedge \hat{\mathrm{H}}+\mathrm{y}^{\mathrm{r}} \sigma\left(\mathrm{a}^{\prime}\right) h s_{1}\right| \hat{\gamma} \wedge \hat{\mathrm{X}}+\mathrm{y}^{\mathrm{r}-1} \mathrm{q}^{-1} \mathrm{ahs}_{1} \mid \hat{\mathrm{H}} \wedge \hat{\mathrm{X}}
$$

without changing its class in $E_{2(r)}^{2,0}$. No element of this form is in the image of $d$, as one can see by looking at the coefficient of $\widehat{\gamma} \wedge \hat{H}$ in (23b), (23c) and (23d), so we can conclude that $E_{2(r)}^{2,0}$ is a free $\mathcal{S}$-module generated by the class of $y^{r+1} h\left|\hat{\gamma} \wedge \hat{A}+y^{r} \sigma\left(a^{\prime}\right) h\right| \hat{\gamma} \wedge \hat{X}+y^{r-1} q^{-1} a h \mid \hat{H} \wedge \hat{X}$. 


\section{References}

[1] L. L. Avramov and S. Iyengar, Gaps in Hochschild cohomology imply smoothness for commutative algebras, Math. Res. Lett. 12 (2005), no. 5-6, 789-804. MR2189239 (2006i:13028)

[2] L. L. Avramov and M. Vigué-Poirrier, Hochschild homology criteria for smoothness, Internat. Math. Res. Notices 1 (1992), 17-25, DOI 10.1155/S1073792892000035. MR1149001 (92m:13020)

[3] V. V. Bavula, Generalized Weyl algebras and their representations, Algebra i Analiz 4 (1992), no. 1, $75-97$ (Russian); English transl., St. Petersburg Math. J. 4 (1993), no. 1, 71-92. MR1171955 (93h:16043)

[4] V. Bavula, Global dimension of generalized Weyl algebras, Representation theory of algebras (Cocoyoc, 1994), CMS Conf. Proc., vol. 18, Amer. Math. Soc., Providence, RI, 1996, pp. 81-107. MR1388045 (97e:16018)

[5] BACH (Buenos Aires Cyclic Homology Group), A Hochschild homology criterium for the smoothness of an algebra, Comment. Math. Helv. 69 (1994), no. 2, 163-168. MR1282365 (95i:16008)

[6] P. A. Bergh and K. Erdmann, Homology and cohomology of quantum complete intersections, Algebra Number Theory 2 (2008), no. 5, 501-522, DOI 10.2140/ant.2008.2.501. MR2429451 (2009h:16010)

[7] P. A. Bergh and D. Madsen, Hochschild homology and global dimension, Bull. Lond. Math. Soc. 41 (2009), no. 3, 473-482, DOI 10.1112/blms/bdp018. MR2506831 (2010e:16014)

[8] R.-O. Buchweitz, E. L. Green, D. Madsen, and Ø. Solberg, Finite Hochschild cohomology without finite global dimension, Math. Res. Lett. 12 (2005), no. 5-6, 805-816. MR2189240 (2006k:16019)

[9] M. A. Farinati, A. Solotar, and M. Suárez-Álvarez, Hochschild homology and cohomology of generalized Weyl algebras, Ann. Inst. Fourier (Grenoble) 53 (2003), no. 2, 465-488 (English, with English and French summaries). MR1990004 (2004d:16014)

[10] Y. Han, Hochschild (co)homology dimension, J. London Math. Soc. (2) 73 (2006), no. 3, 657-668, DOI 10.1112/S002461070602299X. MR2241972 (2007c:16018)

[11] D. Happel, Hochschild cohomology of finite-dimensional algebras, Année (Paris, 1987/1988), Lecture Notes in Math., vol. 1404, Springer, Berlin, 1989, pp. 108-126. MR1035222 (91b:16012)

[12] G. Hochschild, B. Kostant, and A. Rosenberg, Differential forms on regular affine algebras, Trans. Amer. Math. Soc. 102 (1962), 383-408. MR0142598 (26\#167)

[13] L. Richard and A. Solotar, Isomorphisms between quantum generalized Weyl algebras, J. Algebra Appl. 5 (2006), no. 3, 271-285, DOI 10.1142/S0219498806001685. MR2235811 (2007j:16076)

[14] A. G. Rodicio, Smooth algebras and vanishing of Hochschild homology, Comment. Math. Helv. 65 (1990), no. 3, 474-477, DOI 10.1007/BF02566621. MR1069822 (91h:16017)

[15] A. G. Rodicio, Commutative augmented algebras with two vanishing homology modules, Adv. Math. 111 (1995), no. 1, 162165, DOI 10.1006/aima.1995.1019. MR1317386 (96d:16016)

[16] S. P. Smith, A class of algebras similar to the enveloping algebra of sl(2), Trans. Amer. Math. Soc. 322 (1990), no. 1, 285-314, DOI 10.2307/2001532. MR972706 (91b:17013)

[17] A. Solotar and M. Vigué-Poirrier, Two classes of algebras with infinite Hochschild homology, Proc. Amer. Math. Soc. 138 (2010), no. 3, 861-869, DOI 10.1090/S0002-9939-09-10168-5. MR2566552 (2011a:16021)

Departamento de Matemática,

Facultad de Ciencias Exactas y Naturales,

Universidad de Buenos Aires,

Ciudad Universitaria, Pabellón 1

1428, Buenos Aires, Argentina.

Email: asolotar@dm.uba.ar,mariano@dm.uba.ar, qvivas@dm.uba.ar 